\title{
The role of hypoxia and neurogenic genes (Mash-1 and Prox-1) in the developmental programming and maturation of pulmonary neuroendocrine cells in fetal mouse lung
}

\author{
Suzanne McGovern' ${ }^{1}$ Jie Pan ${ }^{1}$, Guillermo Oliver ${ }^{3}$, Ernest Cutz ${ }^{1,2}$ and Herman Yeger ${ }^{1,2}$
}

\begin{abstract}
Pulmonary neuroendocrine cells (PNECs) are the first cell type to differentiate within the primitive airway epithelium, suggesting a possible role in lung development. The differentiation of PNECs in fetal lung is governed by proneural genes such as the mammalian homolog of the achaete-scute complex (Mash-1) and a related transcription factor, hairy and enhancer of split1 (Hes-1). We examined the expression of Mash-1 and a downstream transcription factor Prox-1 in the developing mouse lung of wild-type and respective knockout mouse models. During early stages (embryonic day 12, E12) of development, only some PNECs expressed Mash-1 and Prox-1, but by E15, all PNECs coexpressed both transcription factors. PNECs failed to develop in Mash-1 but not in Prox-1-null mice, indicating that Mash-1 is essential for the initiation of the PNEC phenotype, whereas Prox-1 is associated with the development of this phenotype. As lung develops within a low $\mathrm{O}_{2}$ environment (fetal euoxia, $\mathrm{pO}_{2} \sim 20$ to $30 \mathrm{~mm} \mathrm{Hg}$ ), we examined the effects of hypoxia on PNEC differentiation. Organ cultures of fetal mouse lungs at E12 and E16 were maintained under either $20 \% \mathrm{O}_{2}$ (normoxia, Nox) or $5 \% \mathrm{O}_{2}$ (hypoxia, Hox) and were examined every $24 \mathrm{~h}$ for up to 6 days in culture. In E12 explants, Hox enhanced branching morphogenesis and increased cell proliferation, but PNEC numbers and Mash-1 expression were significantly reduced. This effect could be reversed by switching the explants back to Nox. In contrast, Hox had no apparent effect on Hes-1 expression. Similarly, Hox had no effect on airway branching, PNEC numbers, or Mash-1 expression in E16 explants, indicating locked-in developmental programming. We suggest that during early stages of lung development, $\mathrm{pO}_{2}$ concentration in concert with neurogenic gene expression modulates PNEC phenotype. Thus, disturbances in intrauterine $\mathrm{pO}_{2}$ homeostasis could alter the functional maturation of the PNEC system and hence be involved in the pathogenesis of various perinatal pulmonary disorders.
\end{abstract}

Laboratory Investigation (2010) 90, 180-195; doi:10.1038/labinvest.2009.135; published online 21 December 2009

KEYWORDS: Mash-1; Prox-1; PNEC/NEB development; branching morphogenesis; normoxia/hypoxia

Early stages of lung development are characterized by a continuously branching epithelial tubular system that grows and bifurcates in concert with a surrounding mesenchyme and an expanding vascular supply. However, during the course of lung branching morphogenesis, more that 40 cell types are eventually differentiated, whereas segmentation into trachea, proximal, and distal airways results in a highly specialized organ with complex functions. Within the de- veloping lung, solitary pulmonary neuroendocrine cells (PNECs) are the first cells to differentiate within the primitive airway epithelium, whereas innervated clusters of PNECs forming neuroepithelial bodies (NEBs) develop by midgestation. ${ }^{1}$ PNEC/NEB may have an important role during lung morphogenesis, as these cells secrete a variety of potent bioactive substances, including serotonin or 5HT, and neuropeptides such as gastrin-releasing peptide (GRP) and

\footnotetext{
'Division of Pathology, Department of Paediatric Laboratory Medicine, CIHR Group on Lung Development, The Research Institute, The Hospital for Sick Children, University of Toronto, Toronto, Ontario, Canada; ${ }^{2}$ Department of Laboratory Medicine \& Pathobiology, University of Toronto, Toronto, Ontario, Canada and ${ }^{3}$ Department of Genetics, St. Jude Children's Research Hospital, Memphis, TN, USA

Correspondence: Dr H Yeger, BSc, MSc, PhD, Division of Pathology, Department of Paediatric Laboratory Medicine, The Hospital for Sick Children, 555 University Avenue, Toronto, Ontario, Canada M5G1X8.

E-mail: hermie@sickkids.ca

Received 29 May 2009; revised 9 November 2009; accepted 10 November 2009
} 
calcitonin gene-related peptide (CGRP), which act as local mitogens affecting lung growth and differentiation., ${ }^{2,3}$ The numbers of PNEC/NEB increase toward birth, reaching a peak during the neonatal period. ${ }^{1,4}$ In late fetal and neonatal lung, NEB cells have been shown to express a membranedelimited $\mathrm{O}_{2}$-sensing molecular complex that is activated by hypoxia, leading to the release of 5HT and neuropeptides, suggesting a function as airway oxygen sensors that are important during neonatal adaptation. ${ }^{5-7}$

It is also becoming clear that the lung develops within a hypoxic intrauterine environment (fetal $\mathrm{pO}_{2}$ of 20 $30 \mathrm{~mm} \mathrm{Hg}$ ) and that fetal hypoxia is being increasingly recognized as an important element for intrauterine development. In particular, organogenesis of the kidneys ${ }^{8}$ and cardiovascular system ${ }^{9}$ is dependent on a low-oxygen environment. Recently, hypoxia was shown to increase branching morphogenesis in fetal lung explants. ${ }^{10}$ However, the role of hypoxia in the development and differentiation of PNEC/NEB cells has not been previously investigated.

The molecular mechanisms underlying PNEC/NEB development are only partially known. The mammalian homolog of the proneural gene of the Drosophila achaetescute complex, Mash-1, which encodes a basic helix-loophelix (bHLH)-type transcription factor, has been identified as a key regulatory gene in PNEC development, as Mash-1 knockout mice have no detectable PNECs. ${ }^{11,12}$ Prox-1 (related to the Drosophila homeobox gene, prospero) is involved in the development of taste buds, lens, and the CNS. ${ }^{13-15}$ Both Mash- 1 and Prox- 1 are defined as molecular markers for transient precursors in the CNS. ${ }^{15}$ Prox-1 regulates the exit of progenitor cells from the cell cycle. ${ }^{14} \mathrm{~A}$ more recent study of pre- and postnatal mouse brain showed that Prox-1 was initially expressed in subventricular and early differentiating regions of the brain but was then restricted to the hippocampus and the cerebellum during adulthood, indicating a differential requirement. ${ }^{16}$ The expression of proneural transcription factors Mash-1 and Prox-1 in the nervous system is thus transient and precedes neuronal differentiation. As these two transcription factors are closely associated during development, we investigated the temporal expression of Mash-1 in developing mouse fetal lungs and, along with Prox-1, in early to mid-fetal lung explants, representing two distinct phases in lung development. Furthermore we examined the role of hypoxia on PNEC/NEB differentiation at different stages of the developing lung.

In this study, we show that, whereas Mash-1 specifies initiation of PNEC/NEB differentiation, Prox-1 expression is downstream of Mash-1 and correlates with PNEC/NEB maturation but is not required for NEB morphogenesis. We also show that hypoxia has a critical role in the temporal expression of the PNEC/NEB phenotype. Thus, lung development seems to be orchestrated by gene-environment interactions in which key proneural transcription factors, in concert with oxygenation, pace lung development and phenotypic maturation of airway epithelial cells. The potential significance of our findings in terms of relevance to human disease, especially perinatal lung disorders characterized by PNEC/NEB cell hyperplasia, is discussed.

\section{MATERIALS AND METHODS \\ Mouse Lung Organ Culture}

The animal study protocol was approved by the Animal Research Committee at Hospital for Sick Children. Timedpregnant female C57Bl6 mice were killed at the E12 or E15 stage and their embryos were removed. Plug date was considered day 0 of pregnancy. Lung buds were removed from the embryos in a $\mathrm{CO}_{2}$-independent medium (Gibco, Burlington, ON, Canada) supplemented with penicillin/streptomycin, and these were transferred to porous membranes (cell culture insert, $8 \mu \mathrm{m}$ pore size, $25 \mathrm{~mm}$ diameter; Becton Dickinson Labware, Oakville, ON, Canada) and incubated in six-well culture plates (Becton Dickinson Labware). Three to four explants per membrane were positioned in a well-separated arrangement and cultured at the air-medium interface in a chemically defined medium BGjb (Gibco) containing penicillin/streptomycin (Gibco). The experimental times and conditions used in these studies are shown in Figure 1. Cultures were incubated under $20 \% \mathrm{O}_{2} / 5 \% \mathrm{CO}_{2}$ or $5 \% \mathrm{O}_{2} / 5 \% \mathrm{CO}_{2}$ at $37^{\circ} \mathrm{C}$ for up to 6 days. Half of the culture medium was changed on the third day. In another set of experiments, explants were first exposed to Hox for 6 days and then switched back to Nox for $24 \mathrm{~h}$ (reoxygenation). Control cultures were incubated for the same time period in normoxic conditions. Explants were viewed under phase microscopy with the images being captured using a Nikon digital camera and their layout arranged using Photoshop 7.0.

Pregnant Prox-1 $(+/-)$ mice were provided by Dr G Oliver (St Jude's Children's Research Hospital, Memphis, TN, USA) and because of factors such as transport and timing, only E13 lung explants could be established in culture; however, E12 and E12 lungs were equivalent in terms of PNEC/NEB development. E13 lungs were placed into organ culture as described above for 2 days in normoxic conditions and then harvested for immunofluorescent staining. E13 organs are still considered 'early fetal' and culturing these E13 organs for 2 days was sufficient time to observe neuroendocrine differentiation and allowed us to immunostain for the developmental onset of CGRP (E15 in vivo). Livers from embryos were genotyped to determine wild-type or homozygous Prox1. Blocks of formalin-fixed paraffin-embedded lung tissue from Mash-1(-/-) mouse lung tissues were provided by Dr T Ito (Tokyo, Japan).

\section{Quantification of Branching Morphogenesis}

Branching morphogenesis was quantified under phase microscopy (Olympus BX60) and RSI image software (V.8.0), by counting the number of terminal branches present in explants cultured under normoxic and hypoxic conditions every $24 \mathrm{~h}$ over a 6 -day period. Results were obtained from 

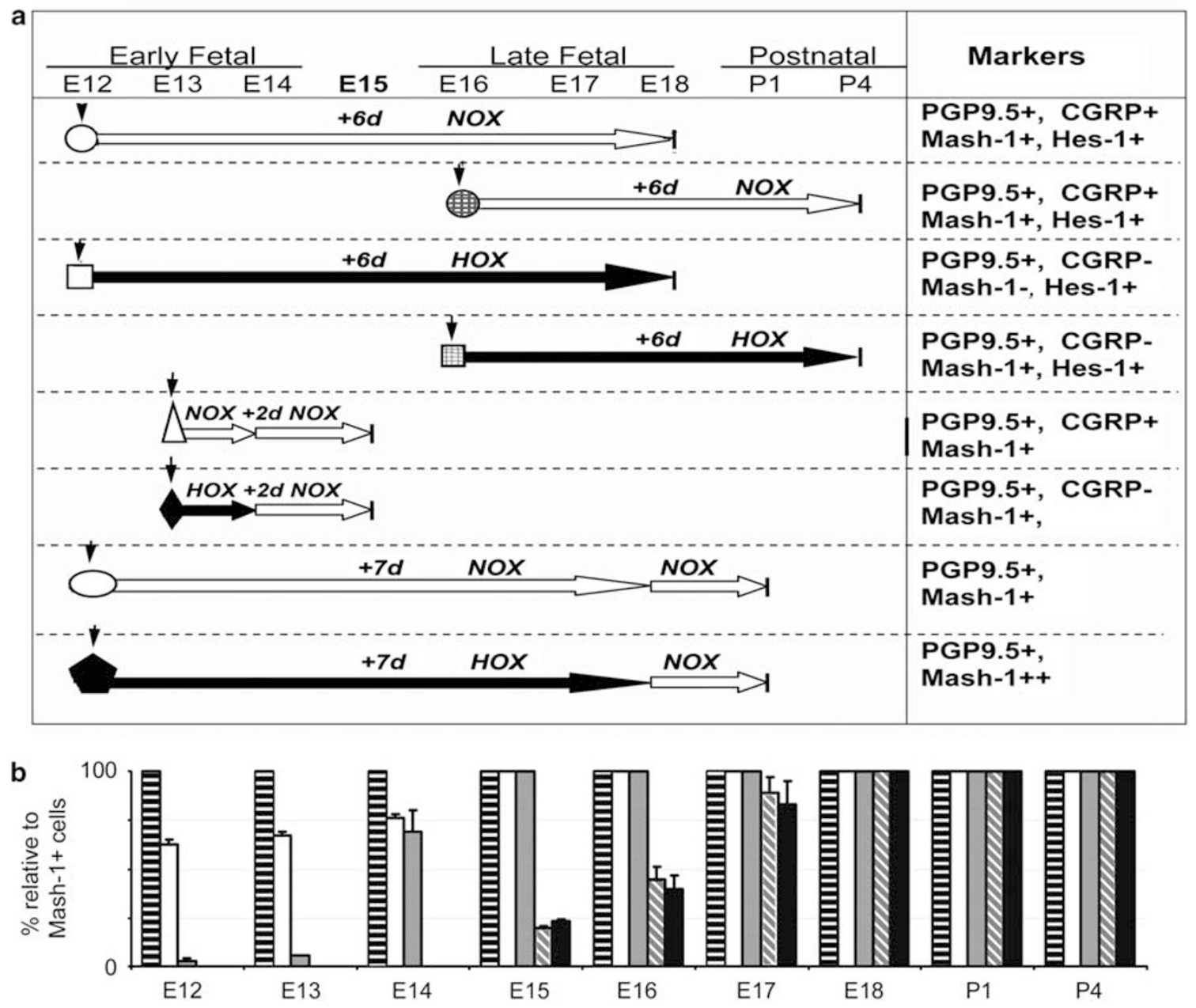

Figure 1 Experimental times, conditions, and summary of results comparing in vivo and explant organ cultures. (a) Experimental times and conditions (normoxia, NOX, large white arrows; hypoxia, HOX, large black arrows) for explant organ cultures are illustrated with respect to gestation period from E12 to P4. Symbols designate manipulations of explants under normoxia and hypoxia. The right-side column summarizes the expressions of neuroendocrine markers with respect to gestational times. Symbols: down arrow, start time of organ culture; circle, E12 +6-day NOX; hatched circle, E16+6-day NOX; square, E12 + 6-day HOX; hatched square, E16 + 6-day HOX; triangle, E12 + 1-day NOX + 1-day NOX; diamond, E12 + 1-day HOX + 1-day NOX; oval, E12 + 6-day NOX + 1-day NOX; pentagon, E12 + 6-day HOX + 1-day NOX. (b) Results are summarized for expressions of these markers in PNEC/NEB relative to Mash-1-positive cells in mouse lungs during this gestational period. Each time point represents morphometric data obtained from five sections (five different pregnancies). A series of sections were immunostained and sections with the maximum number of Mash-1-positive cells were then enumerated for all other markers and expressed as a percentage relative to Mash-1-positive cells. Bars: horizontal black bars, Mash-1; white, Prox-1; gray stippled, PGP9.5; diagonal gray stripes, CGRP; black filled, SV2.

seven different pregnancies $(n=7)$; means \pm s.e. values were calculated and significance was determined by $t$-test (criterion for significance, $P<0.05)$.

\section{BrdU Labeling of Organ Cultures}

Whole-lung E12 organ cultures were maintained for 6 days. Explants were incubated with $10 \mu \mathrm{M} \mathrm{BrdU}$ for $90 \mathrm{~min}$ at $37^{\circ} \mathrm{C}$ for both Nox and Hox conditions. The labeling medium was then removed and fresh medium was added to the tissue for $20 \mathrm{~min}$. The tissue was fixed in zinc formalin (Newcomer Supply, Middletown, WI, USA), washed with PBS, and embedded in O.C.T. (Sakura, Torrance, CA, USA) for frozen sections. Sections were sequentially incubated with $0.05 \%$ trypsin for $20 \mathrm{~min}$, followed by $4 \mathrm{M} \mathrm{HCl}$ for $15 \mathrm{~min}$ at room temperature and then overnight with Mouse on Mouse reagents (Molecular Probes, Eugene, OR, USA) to block nonspecific binding. Subsequently, the sections were incubated with $10 \%$ normal donkey serum for $30 \mathrm{~min}$ at $37^{\circ} \mathrm{C}$ to further block nonspecific antibody binding. Anti-BrdU conjugated with Texas Red (Roche, Laval, QC, Canada) was then added for $45 \mathrm{~min}$ and sections were washed with PBS. DAPI (1:10000) staining was applied to visualize nuclei. BrdU-labeled cells, indicating S-phase, in organ cultures from Nox and Hox conditions were quantified from four consecutive whole-lung sections from each explant $(\mathrm{BrdU}+\mathrm{I}$ DAPI + , both magnification of $\times 100)$. Sections were 
mounted in an antifade medium (Vector Laboratories, Burlingam, CA, USA) and visualized using a fluorescence microscope (Olympus BX60) and RSI image software (V.8.0). Data were analyzed using Sigma Plot to generate figures. Results were obtained from four different pregnancies $(n=4)$ and s.e.m. and a $t$-test (significance if $P<0.05$ ) were used to compare positive BrdU-labeled cells in whole-lung sections (mesenchyme plus airway epithelial) in both Nox and Hox cultures and normalized to DAPI + cells.

\section{Immunohistochemistry/Immunofluorescence Labeling of PNEC/NEB}

Lung buds were dissected from E12 to E15 and fixed in either zinc formalin or Bouins' fixative. After fixation, the tissue was dehydrated through a series of alcohols and embedded in paraffin for immunohistochemistry or snap frozen for immunofluorescence. Organ cultures were processed in a similar manner. Sections $(5 \mu \mathrm{m})$ were deparaffinized and rehydrated through descending alcohol series and in PBS. For antigen retrieval, we used $10 \mathrm{mM}$ sodium citrate buffer $(\mathrm{pH}$ 6.0; Sigma) and endogenous peroxidase quenched with $0.03 \%$ hydrogen peroxide (Fischer) in PBS for $10 \mathrm{~min}$. After blocking nonspecific binding with $10 \%$ secondary species serum, the following primary antibodies and dilutions were used: monoclonals for PGP9.5 (1:400; Ultraclone Ltd, Isle of Wight, England), Mash-1 (1:20; Becton-Dickinson, Oakville, ON, Canada), SV2 (1:100; Iowa Developmental Studies Hybridoma Bank, University of Iowa, Iowa City, IA, USA), and polyclonals for Hes-1 (1:400; gift from Dr T Sudo, Toray Industries, Japan), CGRP (1:400; Chemicon, Temecula, CA, USA), and Prox-1 (1:400; Chemicon). Antibody against CC10 protein (pAb rabbit IgG from Santa Cruz Biotechnology at 1:400 dilution) was used as a marker for Clara cells. For mouse monoclonal antibodies, a Mouse on Mouse blocker (Vector Laboratories) was added to the serum step to reduce nonspecific binding. Specific immunoreactivities were detected using the avidin-biotin complex method (Vectastain ABC kit; Vector Laboratories) and biotinylated secondary antibody, followed by avidinTexas Red (Jackson Laboratories, West Grove, PA, USA), as previously reported. ${ }^{17}$ Peroxidase was visualized with $\mathrm{DAB}$ chromogen (Invitrogen, Burlington, ON, Canada) and counterstained with hematoxylin. As a negative control, the primary antibody was omitted. For immunofluorescence, tissue was fixed in a solution of zinc formalin and frozen sections were prepared. Visualization of PGP9.5, CGRP, and SV2 was carried out using an indirect immunofluorescence method using either FITC- and Texas Red-conjugated secondary antibodies. Omission of the primary antibody did not show labeling above background levels in the lung airway epithelium. Immunofluorescence-labeled tissues were analyzed using confocal microscopy as previously described ${ }^{17}$ or an Olympus Microscope BX60, and were visualized with RSI image software (V.8.0).

\section{Genotyping Prox-1 Mice}

Cell lysates were prepared from individual E13 fetal livers and from the liver of the heterozygous mother. For PCR amplification, we used $50 \mu \mathrm{l}$ of buffer containing each dNTP at $50 \mathrm{mM}, 6 \mathrm{pmol}$ of each primer, $50 \mathrm{mM}$ Tris- $\mathrm{HCl}(\mathrm{pH} 8.5)$, $1.5 \mathrm{mM} \mathrm{MgCl}_{2}, 20 \mathrm{mM}\left(\mathrm{NH}_{4}\right)_{2} \mathrm{SO}_{4}, 10 \%$ dimethyl sulfoxide, and $0.2 \mathrm{U}$ of Taq polymerase. Initial denaturation was for $3 \mathrm{~min}$ at a temperature of $94^{\circ} \mathrm{C}$, followed by 35 cycles. Annealing was set for $45 \mathrm{~s}$ at $60^{\circ} \mathrm{C}$, followed by $45 \mathrm{~s}$ of polymerization at $72^{\circ} \mathrm{C}$ and denaturation for $45 \mathrm{~s}$ at $94^{\circ} \mathrm{C}$. The cycle was repeated 35 times. The primer sequence used was Wt Fwd: 5'-ATCGCTTATGTGATGAGCACC-3'; Wt Rev: 5'-CCGAGTCCTGGGCCCGTGCAT-3'; and Mut Fwd: 5'GAAGCGGGAAGGGACTGGCTGCTA-3'; Mut Rev: 5'-CGG GAGCGGCGATACCGTAAAGC-3'.

\section{RNA Isolation and RT-PCR}

For all mouse tissues, $\sim 5 \mathrm{mg}$ of tissues for each sample was sonicated in TRIzol solution (Invitrogen, Carlsbad, CA, USA), homogenized by ultrasonicator, and applied to RNA purification columns according to the manufacturer's instructions (SupperArray, Frederick, MD). After washing the columns, the bound RNA was treated with DNAse I, washed, and eluted. A volume of $200 \mathrm{ng}$ of total RNA (from each tissue sample) was reverse transcribed with RT annealing mixture in an iScript Select cDNA Synthesis Kit (Bio-Rad, Hercules, CA) and PCR was performed. The primer pairs used were Mash1: Fwd, 5'-AATGGACTTTGGAAGCAGGA3'; and Rev, 5'-TTAGGTTCAGACACCAGTCAC-3'; Hes1: Fwd, 5'-CACAGAAAGTCATCAAAGCC- $3^{\prime}$ and Rev, 5'TGCTTCACAGTCATTTCCAG- $3^{\prime}$. Controls included mouse E18 brain, which expresses abundant Mash1 mRNA, and mouse E18 liver, which is negative for Mash1 and positive for Hes1. For normalization of total RNA, a primer pair representing mouse $\beta$-actin (Clontech, Palo Alto, CA, USA) was included in the same reactions. Amplified products and DNA molecular weight markers (100 bp DNA Ladder; Invitrogen, Burlington, ON, Canada) were separated by electrophoresis in $2 \%$ agarose gels.

\section{Immunoprecipitation and Western Blots}

For nuclear protein preparation, lungs from different developmental stages (embryonic and newborn mouse) and lung explant cultures from various experiments were dissociated with strong vortexing in a hypotonic HEPES solution with $1 \times$ protease inhibitor cocktail (Pierce). Lysates were centrifuged at $1000 \mathrm{~g}$ for $10 \mathrm{~min}$ at $4^{\circ} \mathrm{C}$ to remove the supernatant containing cell membranes and soluble cytoplasm proteins, and nuclear pellets were resuspended in RIPA solution and sonicated on ice with $1 \times$ protease inhibitor cocktail. Protein concentrations were measured using the $D C$-Bio-Red protein detection kit (Bio-Rad Laboratories, Hercules, CA, USA).

For immunoprecipitation and western blots, $200 \mu \mathrm{g}$ of each protein sample was coimmunoprecipitated with either 
Mash-1/ $\beta$-tubulin or Hes-1/ $\beta$-tubulin antibodies using the Seize $X$ Protein $G$ Immunoprecipitation Kit from Pierce(Thermo Fisher Scientific, Rockford, IL, USA), following the manufacturer's instructions. A volume of $20 \mu \mathrm{l}$ of samples was mixed with $2 \times$ sample buffer. After boiling at $100^{\circ} \mathrm{C}$ for $5 \mathrm{~min}, 30 \mu \mathrm{l} / \mathrm{lane}$ of the precipitated nuclear protein solution and $10 \mu \mathrm{l}$ of Odyssey Fluo-protein marker (Li-COR, Lincoln, NE, USA) were electrophoresed in a 10\% SDS-PAGE with $3 \%$ stacking gel and then electroblotted onto PVDF membranes using a Bio-Rad Mini-Gel Transfer system. After blocking with $4 \%$ BSA $+1 \%$ skim milk, membranes were processed through overnight coincubations with mouse monoclonal anti-Mash- 1 and mouse anti- $\beta$-tubulin antibodies or with rabbit anti-Hes- 1 and mouse anti- $\beta$-tubulin antibodies at $4^{\circ} \mathrm{C}$. After washing in TBS-Tween 20 , membranes were incubated for $1 \mathrm{~h}$ at room temperature in Qdot Secondary Antibody Conjugates (Qdot705 goat anti-mouse IgG (red)) that were diluted at 1:1000 in blocking buffer. After final washing in TBS-Tween 20, membranes were analyzed immediately using the Image Station 2000MM system with Filtered Epi Illumination (KODAK 415/100 bp) and images were captured using a CCD camera. Band densities within the linear range were analyzed with NIH Image J software. Values were obtained for individual samples (three separate scans performed in duplicate) and normalized to the mean values of $\beta$-tubulin used as housekeeping protein on the same blot with the same exposure time. Mean and s.e. values were calculated for each lane $(n=3)$. The nonparametric, unpaired $t$-test was used for comparison between the two groups with Prism GraphPad version 5.01 software (GraphPad Prism Software).

\section{Quantification of PNECs}

Analysis of PGP9.5-positive PNECs per $\mathrm{mm}^{2}$ tissue was carried out by manually counting individual PNECs per section (minimum of four sections/lung) as normalized to the individual lung bud area using an Olympus microscope and SimplePCI software on acquired digital images. As it was difficult to discriminate different lung areas in explants, PNECs were enumerated per total explant area. The information displayed in each graph represents total counts and comprised different numbers of lungs ( $n$ values) and pregnancies. For Figure $4 \mathrm{~g}$ and $\mathrm{h}$, data were obtained from $n=7$ and $n=4$, and seven and four different pregnancies, respectively. For Figure 5, data were obtained from $n=5$ and five different pregnancies. Standard error of the mean with a $P<0.05$ ( $t$-test) was used to determine the level of significance.

\section{RESULTS \\ Developmental Profile of PNECs in Mouse Lung: Mash-1 and Prox-1 Identify an Airway Epithelial Cell Sub- population Committed to the PNEC/NEB Phenotype}

Immunolabeling for Mash-1 and Prox-1 was individually performed on paraffin sections of lung from mice at E12, E13, E14, and E15 stages of development. Both Mash-1 and Prox-1 immunoreactivity was localized to the nuclei of single or clusters of primitive airway epithelial cells distributed along the basement membrane (Figure 2a-h). Often Mash-1and Prox-1-positive cells were arranged in rows, especially at airway branch points, the preferential location for NEB in mature lungs (Figures $1 \mathrm{f}$ and $2 \mathrm{~b}$ ). In general, most cells expressing Mash-1 at E12 and E13 were negative for Prox-1 (Figure $2 \mathrm{~b}$ versus $\mathrm{f}$ ); however, in some areas, there was coexpression of Mash-1 and Prox-1 in nascent NEB (Figure 2a versus e, and b versus f). In comparison, by E15, all Mash-1positive cells also became Prox-1 positive (Figures $1 \mathrm{~h}$ and $2 \mathrm{~d}$ ). For colocalization of both Mash-1 and Prox-1 in the same lung sections, we used double immunofluorescence labeling and confocal microscopy (Figure 2i-1). Between E12 and E14, about half of the Mash-1-positive cells were also Prox-1 immunoreactive, whereas at E15, all Mash-1-positive cells expressed Prox-1 (Figure 2j-1). Morphometric analysis showed a progressive and nearly linear increase in Mash-1 and Prox- 1 coexpressing cells from about $60 \%$ at E12 to $100 \%$ by E15. The results of these studies are summarized in Figure 1.

The development and maturation of PNEC/NEB cells in the lung occurs in a proximal to distal direction. ${ }^{18}$ Consequently, PNEC/NEB cells develop first in the proximal better-

\footnotetext{
Figure 2 Expression of Mash-1 and Prox-1 in the developing mouse lung. Comparison of immunolocalization of (a-d) Mash-1 and (e-h) Prox-1 on adjacent paraffin sections from E12 to E15 mouse lung. Arrows point to positively stained colocalization of Mash-1 and Prox-1 in PNEC/NEB cells distributed along the airway epithelial basement membrane. The airway in panels $\mathbf{a}$ and $\mathbf{e}$ shows coexpression in PNEC/NEB, but as more evident in panels $\mathbf{b}$ and $\mathbf{f}$, many Mash-1positive cells did not express Prox-1 (original magnification $\times 200$ ). (i-I) Confocal microscopy of dual immunofluorescence labeling for Mash-1 (red signal) and Prox-1 (green signal) on frozen sections of mouse lungs between E12 and E14. During early stages of lung development (i-k), only some Mash-1 + cells also coexpressed Prox-1 + (orange signal, arrow, as in panel k). However, by E15, all Mash-1 + cells were also Prox-1 + (I) (original magnification $\times 480$ ). Higher magnification views of NEB in E14 $(\mathbf{m}, \mathbf{n})$ and E15 $(\mathbf{o}, \mathbf{p})$ mouse lungs with dual immunoflorescence for PGP9.5/Mash-1 and Mash-1/Prox-1. (m) Dual immunolabeling for Mash-1 in nuclei (red signal) and for neuroendocrine marker PGP9.5 (green signal) in the cytoplasm of NEB cells residing in a larger airway that is better differentiated. (n) Coexpression of Mash-1 and Prox-1 (orange signal) in nuclei of NEB cells located at airway bifurcation. (o) NEB cells showing localization of PGP9.5 (green signal) in the cytoplasm and developing submucosal nerve fibers entering the NEB base, whereas Mash-1 (red signal) is localized in nuclei. (p) A large NEB cell cluster with a uniform coexpression of Mash-1 and Prox-1 (orange signal) in all nuclei. (o) Note fine nerve fibers (arrow) underneath the NEB that label for PGP9.5 (original magnification $\times 640$ ). (q) Western blot and (r) RT-PCR analyses of gestational series of lungs (E11E18 and P4). Expression of Mash-1 protein and mRNA peaks at E15 correlating with the immunostaining shown above. No changes were noted in Hes-1 mRNA or protein expression during the same gestation period, except for an apparent increase at P4. Densitometry of western blots confirms a gradual increase in Mash-1 protein expression, peaking at E15 and then decreasing by P4 with minimal variation for Hes-1. All values are relative to positive controls for Mash-1 (brain) and Hes-1 (liver). Loading controls are tubulin for western blots and actin for RT-PCR.
} 
differentiated airways, whereas in more peripheral primitive tubules, the immature pulmonary epithelium lacks PNEC/ NEB and seems diffusely PGP9.5 positive. ${ }^{19}$ We therefore examined the coexpression of PGP9.5, a neuroendocrine marker, with Mash-1 and Prox-1 using double immunofluorescence labeling. At the E14 stage of development, in the larger more proximal airways, single and small clusters of
Mash-1-positive cells were PGP9.5 positive, and well-defined NEB coexpressed Prox-1 (Figure $2 \mathrm{~m}$ and $\mathrm{n}$ ). In comparison, in the small, undifferentiated airways, both epithelium and PNECs were PGP9.5 positive (see Figure 3a). At E15, all Mash-1-positive cells were Prox-1 and PGP9.5 positive, including nascent submucosal nerve fibers innervating NEB (Figure $2 \mathrm{o}$ and $\mathrm{p}$ ). Thus, E15 is seen as an important de-
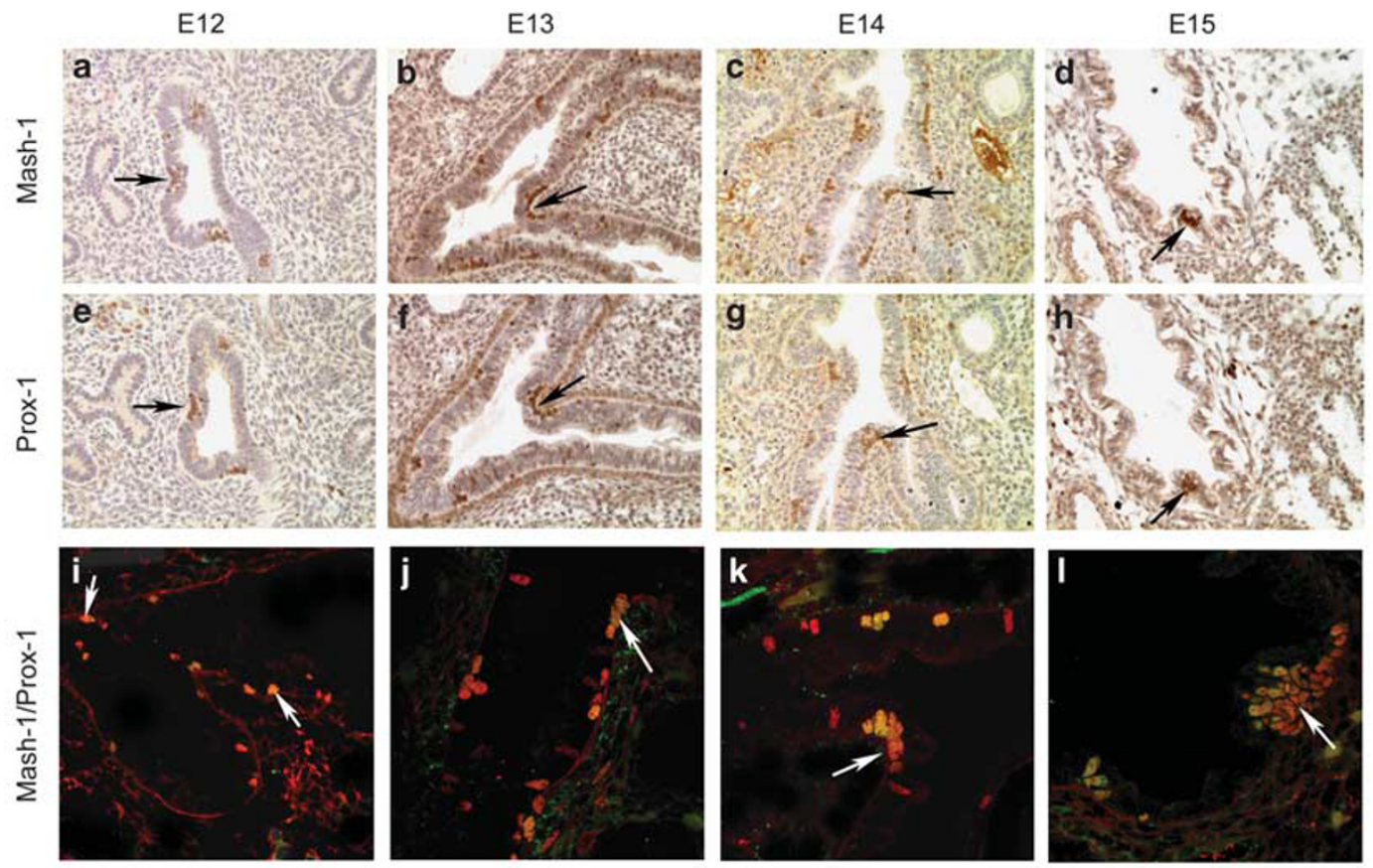

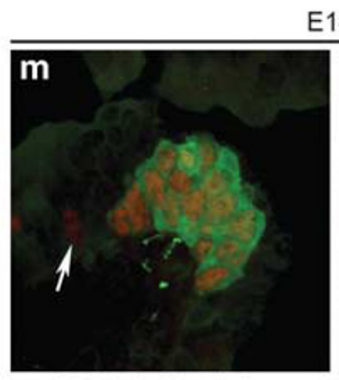

PGP9.5+Mash-1
14

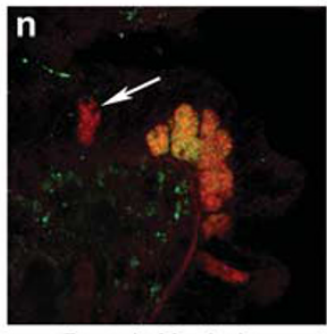

Prox-1+Mash-1

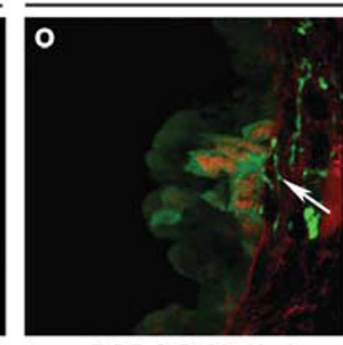

PGP 9.5+Mash-1

E15

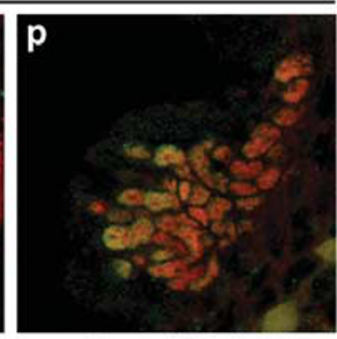

Prox-1+Mash-1

q

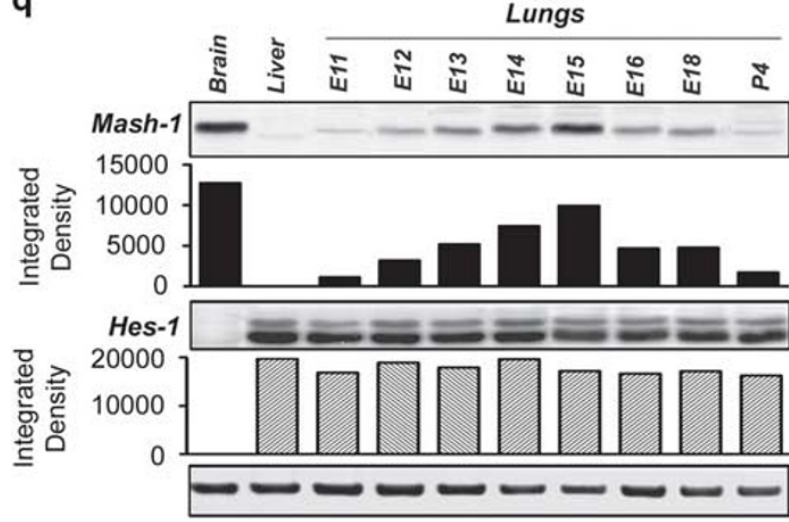

$\mathbf{r}$

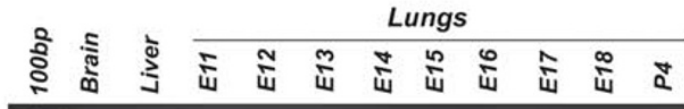

Mash-1

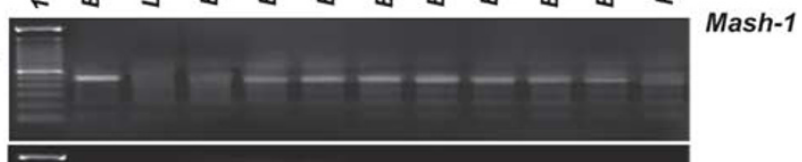

Hes-1

Tubulin

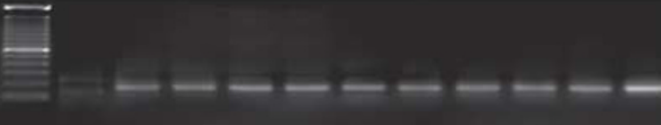



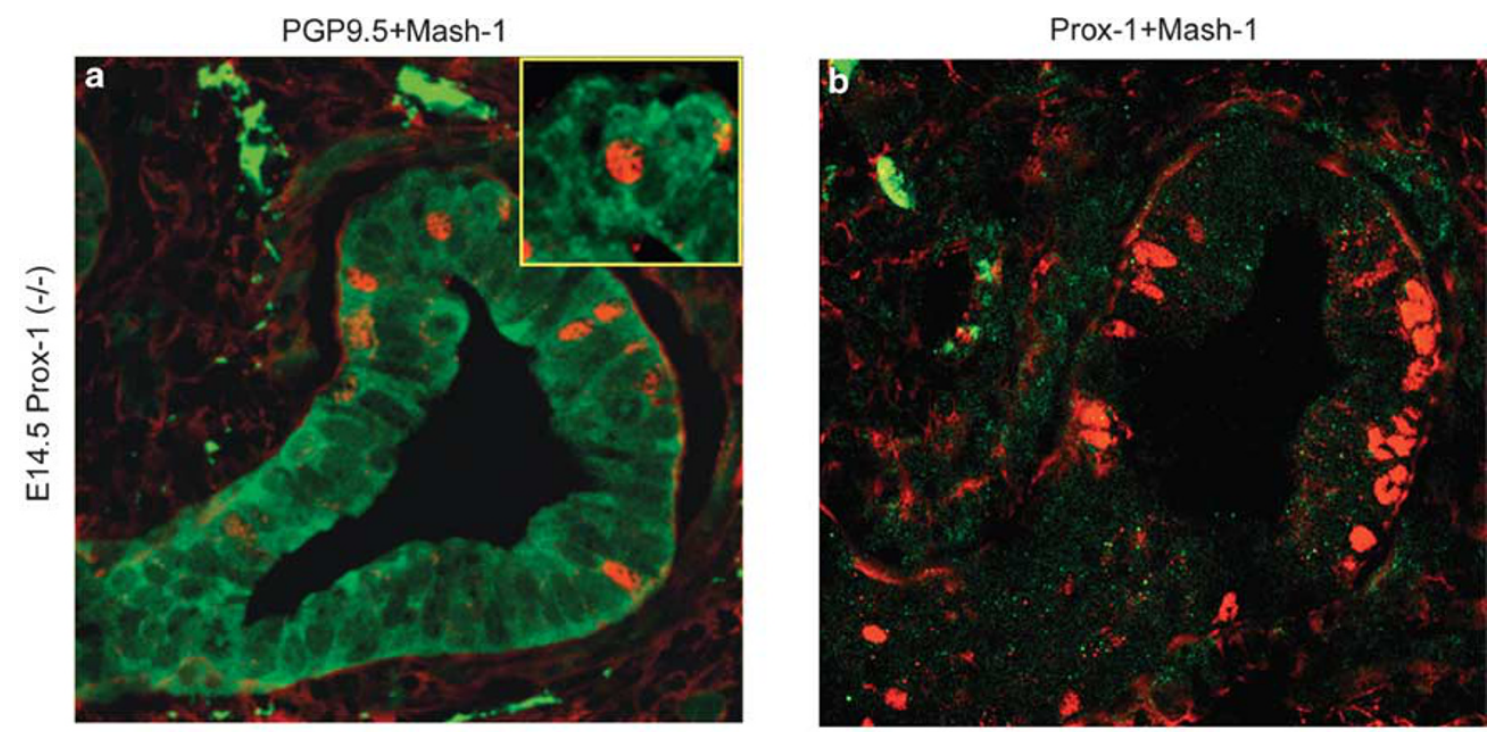

PGP9.5+Mash-1

\section{CGRP+Mash-1}
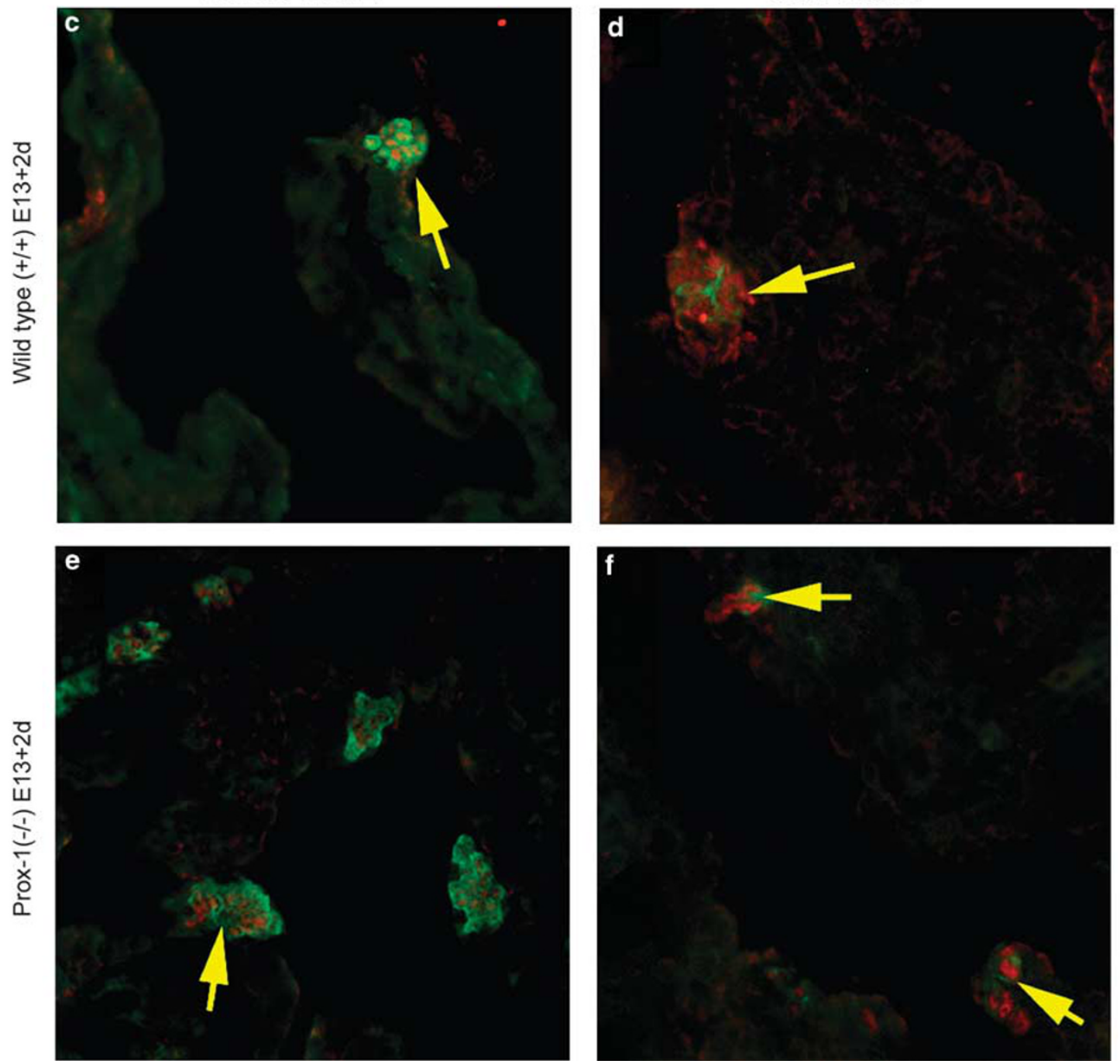
velopmental transition point for acquisition of the neuroendocrine phenotype. Taken together, immunolabeling studies indicate that Mash-1 expression precedes Prox-1 and PGP9.5 expression in PNEC/NEB cells (Figure 1).

The findings of developmental regulation of Mash-1 expression observed by immunohistochemistry were confirmed by western blot analysis (Figure 2q) and RT-PCR (Figure 2r) of lung tissue extracts from fetal stages of E12-E18 and postnatally at day 4 (P4). Developmental expression of Mash1 that peaked at E15 correlated well with the immunolabeling findings. In contrast, Hes-1 expression, a marker of the nonneuroendocrine respiratory epithelium, did not change throughout fetal lung development, again correlating with the immunolabeling observations (Figure $2 \mathrm{q}$ and $\mathrm{r}$ ). An apparent increase in Hes-1 at P4 is suggestive of respiratory epithelial maturation.

\section{PNEC Expression and Differentiation in Mash-1- and Prox-1-Null Mice}

To further explore the roles of Mash-1 and Prox-1 in PNEC/ NEB development, we examined mutant mouse strains in which Mash-1 and Prox-1 expressions were genetically disrupted. As expected, paraffin sections of lung from Mash-1 knockout (-I-) mice on P3 showed an absence of Mash-1positive cells and lack of demonstrable PNEC/NEB, as determined by the absence of PGP9.5- and CGRP-expressing cells in the airway epithelium (data not shown). Serial sections immunostained for Prox-1 also showed an absence of Prox-1-positive cells, suggesting that expressions of these genes were linked. Conversely, we next studied PNEC/NEB in the lungs of Prox-1 mutant mice. As Prox-1(-/-) mice survive only until E14.5, we examined frozen sections of lung from these mice immunostained for Mash-1 and found cells within the airway epithelium that showed Mash-1-positive nuclei, but their cytoplasm was negative for PGP9.5 (Figure $3 \mathrm{a}$ and $\mathrm{b}$ ). This finding suggests that Mash-1-committed undifferentiated PNEC/NEB cells are present in the lungs of Prox-1(-/-) mice. However, on first analysis, Prox-1 expression seemed to be linked to the development of the PNEC/NEB neuroendocrine phenotype, as these Mash-1positive cells did not express PGP9.5. However, when lung explants from E13 Prox $(-/-)$ lungs were maintained in culture for 2 days, we found Mash1-positive cells that expressed both PGP9.5 and CGRP, suggesting that PNEC maturation can proceed in the absence of Prox- 1 as long as Mash-1 expression is maintained (Figure $3 \mathrm{c}-\mathrm{f}$ ). There was no significant difference in the number and frequency of PGP9.5-positive PNEC/NEB cells in the lungs of wild-type compared with Prox-1(-/-) mice.

\section{The Effects of Hypoxia on Epithelial Branching Morphogenesis, Cell Proliferation, and PNEC/NEB Differentiation}

To study the effects of hypoxia on lung development and PNEC/NEB differentiation, we used organ cultures of lung explants maintained on culture inserts and exposed either to normoxia $\left(20 \% \mathrm{O}_{2}\right)$ or hypoxia $\left(5 \% \mathrm{O}_{2}\right)$. E12 lung buds were explanted under normoxic and hypoxic conditions and peripheral branching (terminal ends) was assessed every $24 \mathrm{~h}$ over a 6-day period. Over a 6-day culture period, lung explants underwent exponential growth, and complexity of the distal airways became evident (Figure $4 \mathrm{a}, \mathrm{c}$ and e). In agreement with previous reports, ${ }^{10}$ organ cultures exposed to hypoxia had significantly more terminal branches compared with parallel cultures grown in normoxic conditions ${ }^{10}$ (Figure $4 \mathrm{~b}, \mathrm{~d}$ and $\mathrm{f}$ ). A quantitative study of peripheral bud counts showed a significant increase in airway branching under hypoxia compared with normoxia (Figure $4 \mathrm{~g}$ ). Organ cultures exposed to hypoxia showed a higher proliferative index $(\sim 49 \%)$ compared with normoxia $(\sim 33 \%)$ using the BrdU labeling method (Figure 4h).

To examine the effects of hypoxia on PNEC/NEB differentiation, some E12 explants were maintained in hypoxia for $24 \mathrm{~h}$ (short-term hypoxia), and others for 6 days (long-term hypoxia), and then immunostained for PGP9.5 and SV2 (Figure 5) Control lung explants cultured under normoxia underwent extensive PNEC differentiation, as indicated by the presence of numerous PGP9.5- and SV2-positive PNEC/ NEB cells and the development of large corpuscular NEB cells (Figure 5a and $\mathrm{c}$ ). In contrast, lung explants maintained under hypoxia showed a markedly reduced expression of both PGP9.5- and SV2-positive cells in the airway epithelium (Figure 5b and d), although bronchial ganglion cells and nerves immunoreactive for PGP9.5 and SV2 were unaffected (data not shown). These effects of hypoxia seemed to be PNEC/NEB-cell-specific, as no changes were observed in the expression or frequency of CC10-positive epithelial cells corresponding to differentiating Clara cells (Figure 5e and f). Morphometric analysis showed more than a fivefold reduction in the number of PGP9.5 immunoreactive PNEC/NEB cells in explants maintained in hypoxia compared with control normoxia cultures (Figure 5g).

Figure 3 Immunofluorescence labeling of Prox-1(-/-) lungs in situ at E14.5 (a, b) and in lungs from E13 after 2 days in explant culture (c-f). (a) Prox-1(-/-) E14.5 lung showing weak diffuse immunostaining for PGP9.5 in the cytoplasm of epithelial cells from a smaller distal airway including PNEC/NEB (green signal), with positive Mash-1 expression (red signal) in nuclei. Inset: higher magnification of a Mash-1-positive cell with background PGP9.5 staining. (b) The Prox-1(-/-) E14.5 lung dual immunostained for Mash-1 (red signal) showing positive NEB cell nuclei, whereas immunolabeling for Prox-1 (green signal) is negative (original magnification $\times 480)$. Wild-type $(\mathbf{c}, \mathbf{d})$ and Prox-1(-/-) (e, $\mathbf{f})$ embryonic lungs grown in organ culture showing appropriate expression of PNEC/NEB cell cytoplasmic markers (green signal) PGP9.5 (c, e) and CGRP (d, f), whereas their nuclei are Mash-1 (red signal) positive. Arrows point to NEB (original magnification $\times 400$ ). 

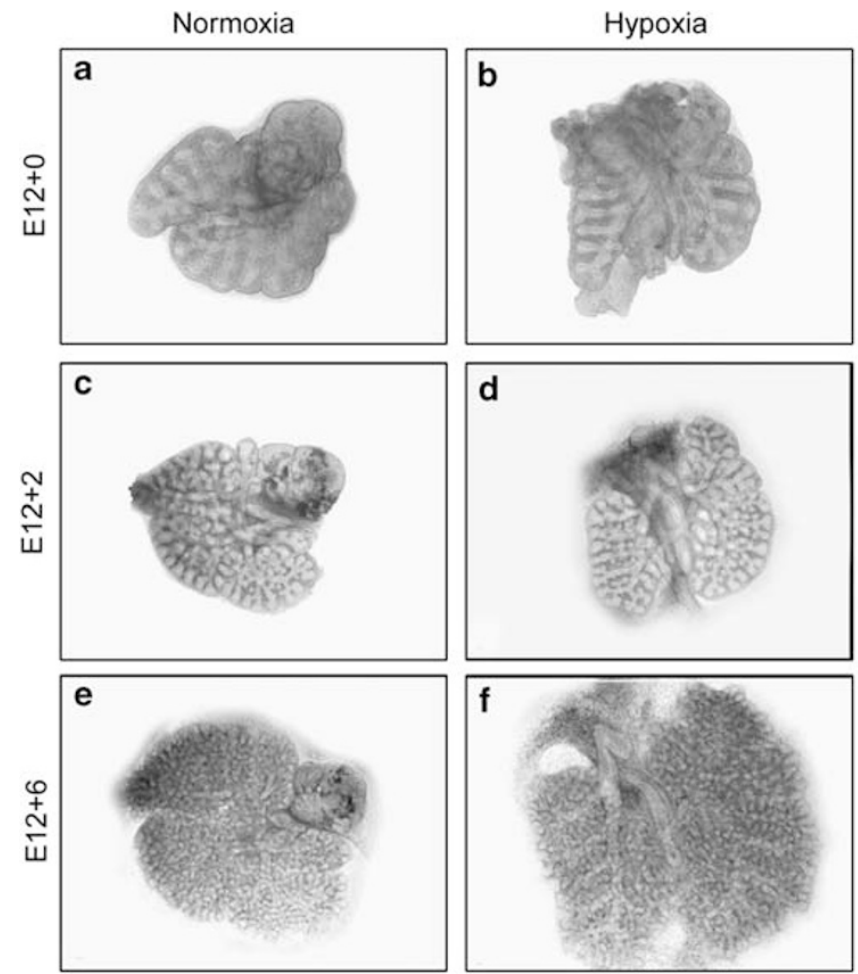
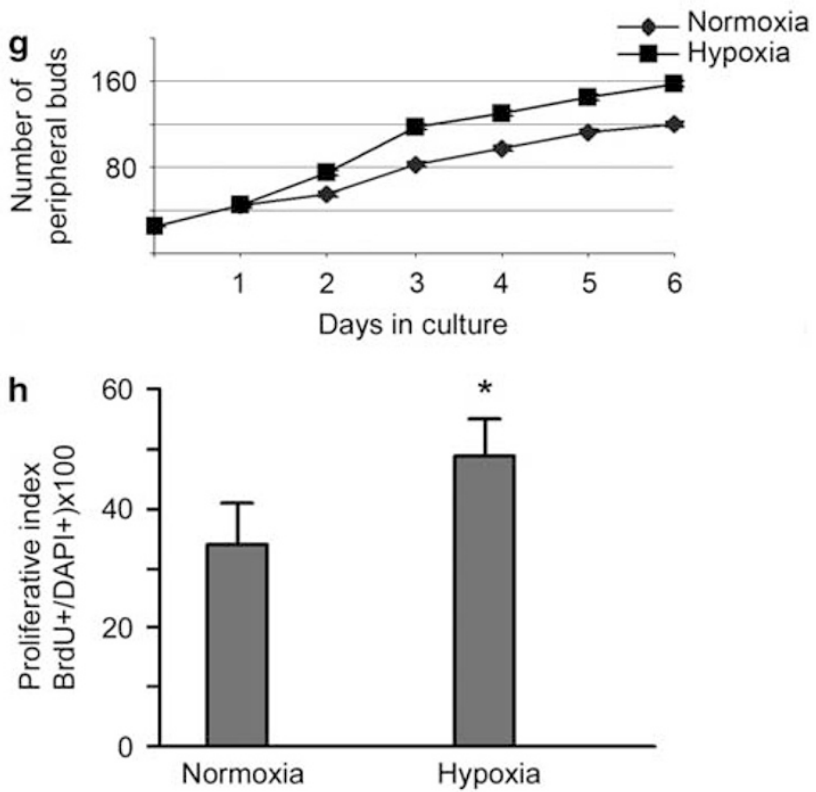

Figure 4 Long-term ( 6 days) explant organ culture of E12 lungs evaluated for branching morphogenesis and cell proliferation. Gross appearance of lung explants maintained in either normoxia (a, c, and e) or hypoxia (b, d, and $\mathbf{f}$ ). Size of lungs increased more than twofold after 6 days in hypoxia (f) compared with normoxia (e). (g) Morphometric $(n=7)$ assessment of branching morphogenesis showed a significant and sustained increase in airway branching after 3 days in hypoxia compared with cultures maintained under normoxia. (h) Proliferative index (assessed by BrdU labeling; $n=4$ ) of lung E12 organ cultures exposed to 6 days of hypoxia is significantly increased compared with normoxia $\left({ }^{\star} P<0.05\right)$.

E12 explants exposed to hypoxia for 6 days showed a markedly reduced expression of Mash-1 compared with normoxia control (Figure 6a and b), whereas that of Hes-1, a marker for the nonneuroendocrine epithelium, was maintained (Figure $6 \mathrm{c}$ and $\mathrm{d}$ ). Thus, the nonneuroendocrine airway epithelial cell component seemed to be unaffected by hypoxia. In lung samples taken after stage E15, PNEC/NEB cells became insensitive to hypoxia, as E16 lung explants cultured for 6 days under hypoxia retained a full expression of both Mash-1 and Hes-1, comparable to that of normoxia controls (Figures 1 and $6 \mathrm{~g}-\mathrm{j}$ ).

It is evident from the data in Figure 1 that PNEC/NEB maturation is initiated at $\mathrm{E} 15$ and that, together with the in vitro observations, a critical mid-gestation switch occurs in the hypoxia-sensitive to hypoxia-insensitive developmental phase corresponding to maturation of PNEC/NEB cells after E15. These observations support the notion that E15 is a critical time point in PNEC/NEB development when they become refractory to the hypoxia stimulus.

\section{Reoxygenation (Normoxia) Restores Mash-1 Expression in PNEC/NEB Turned Off by Exposure to Hypoxia}

As we found that hypoxia downregulated Mash-1 expression, we asked whether Mash-1 expression is possibly regulated through oxygenation. In these experiments, E13 explants were first placed under hypoxia for 1-6 days and then switched back to normoxia for a 24 -h (1 day) period. As expected, in explants exposed to hypoxia (both short- and long term), Mash-1 expression was significantly reduced but became re-expressed strongly when lung explants were switched to normoxia (Figure $7 \mathrm{a}-\mathrm{h}$ ). Although in control lung explants maintained under normoxia PNEC/NEB cells showed positive CGRP immunoreactivity (Figure 7b), it was negative in the lung explants that were switched from hypoxia to normoxia (Figure 7d). In comparison, in the longterm cultures switched from hypoxia to normoxia (Figure 7h), expression of PGP9.5 was increased, coincident with a strong expression of Mash-1. These observations suggest that hypoxia can acutely regulate the differentiation of PNEC/ NEB and thus pace this developmental process. In addition, as there was a more robust expression of Mash-1 after reoxygenation when lung explants were switched from hypoxia to normoxia, this observation suggested that $\mathrm{pO}_{2}$ concentration may be important for the expansion of Mash-1committed PNEC progenitors in which Mash-1 expression precedes maturation markers such as CGRP and seems to coincide with the induction of PGP9.5 expression.

To provide quantitative assessment of the expression of Mash-1 and Hes-1 at the mRNA and protein level, respectively, we performed RT-PCR and western analysis of extracts 

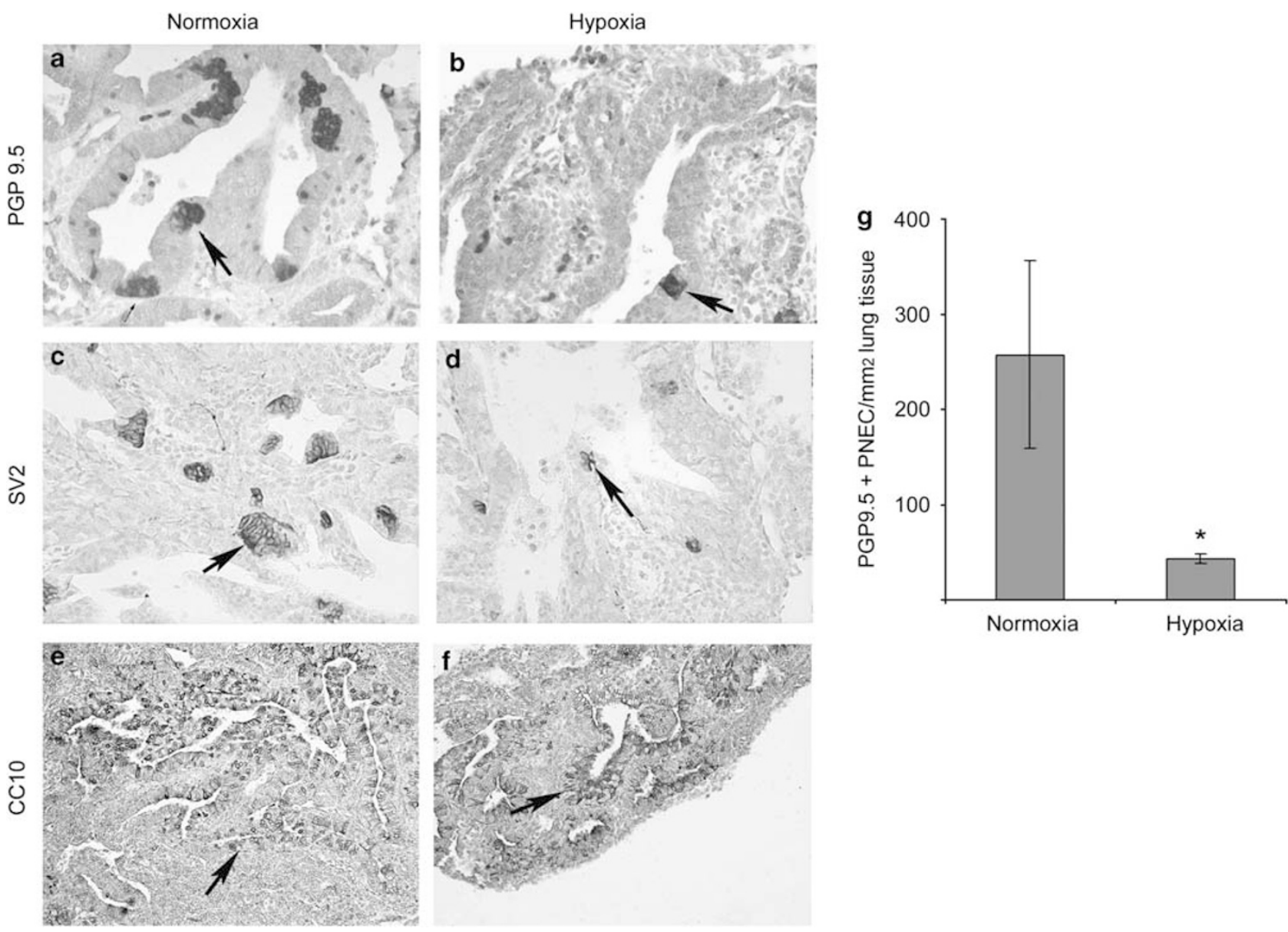

Normoxia

Hypoxia

Figure 5 Immunohistochemical labeling of PNEC/NEB cells (a-d) and Clara cells $(\mathbf{e}, \mathbf{f})$ in E12 lung organ cultures maintained in normoxia or hypoxia for 6 days. In normoxia cultures $(\mathbf{a}, \mathbf{c}$ ), the size and number of PNEC/NEB cells (arrow) immunostained for PGP9.5 and neuroendocrine marker SV2 were markedly increased compared with that of cultures maintained in hypoxia (b, d) (original magnification $\times 400$ ). Expression of Clara cell marker CC10 in airway epithelial cells (arrow) was comparable between cultures exposed to normoxia (e) or hypoxia (f) (original magnification $\times 200$ ). (g) Quantification of immunoreactive PNEC/NEB cells per $\mathrm{mm}^{2}$ of lung tissue $(n=5)$ confirmed significant reduction $\left({ }^{*} P<0.05\right)$ in these cells in lung explants maintained under hypoxia compared with those under normoxia.

from lung explants under different $\mathrm{pO}_{2}$ conditions. Owing to the relatively low numbers of PNEC/NEB cells in the lung (representing less than $1 \%$ of lung epithelial cells), we used immunoprecipitation to concentrate Mash-1 protein and a highly sensitive detection system for western blot as described in Materials and Methods. As expected, in E12 lung explants maintained in hypoxia for 6 days, the expression of both Mash-1 mRNA and protein was significantly reduced, whereas Hes-1 expression was unaffected (Figure 8a and b). Again, when explants exposed to hypoxia were switched to normoxia for $24 \mathrm{~h}$, there was a robust upregulation of Mash-1 mRNA and protein expression, supporting the notion that hypoxia has a regulatory role in Mash-1 expression and thereby PNEC/NEB development. Significantly, in E16 explant cultures, hypoxia had no effect on Mash-1 expression, in agreement with immunostaining data (Figure $8 \mathrm{a}$ and b), highlighting this critical developmental switch at E15.

\section{DISCUSSION}

The principal aim of this study was to investigate the role of neurogenic genes in the differentiation of PNEC/NEB cell lineage in the developing lung and to examine the effects of different $\mathrm{pO}_{2}$ concentrations on this process. Our studies (summarized in Figure 1) suggest that the bHLH-type transcription factor, Mash-1, and the homeodomain-containing protein, Prox-1, not only delineate a transient precursor of PNEC/NEB cells but also define the temporal limits between the process of initiation and maturation. Previous studies have identified Mash-1 as the obligatory transcription factor required for the development of PNEC/NEB, as Mash-1-/mouse lungs are devoid of these cells. ${ }^{12}$ In this study, we show that Mash-1 remains the earliest known expression marker specific to PNEC/NEB and that Prox-1 is involved in the subsequent specification of PNEC/NEB phenotypic maturation. 


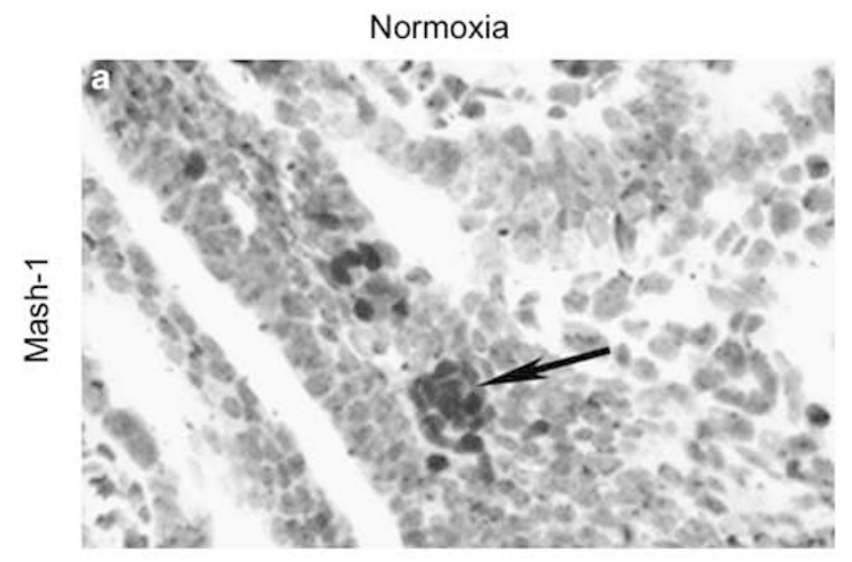

Hypoxia
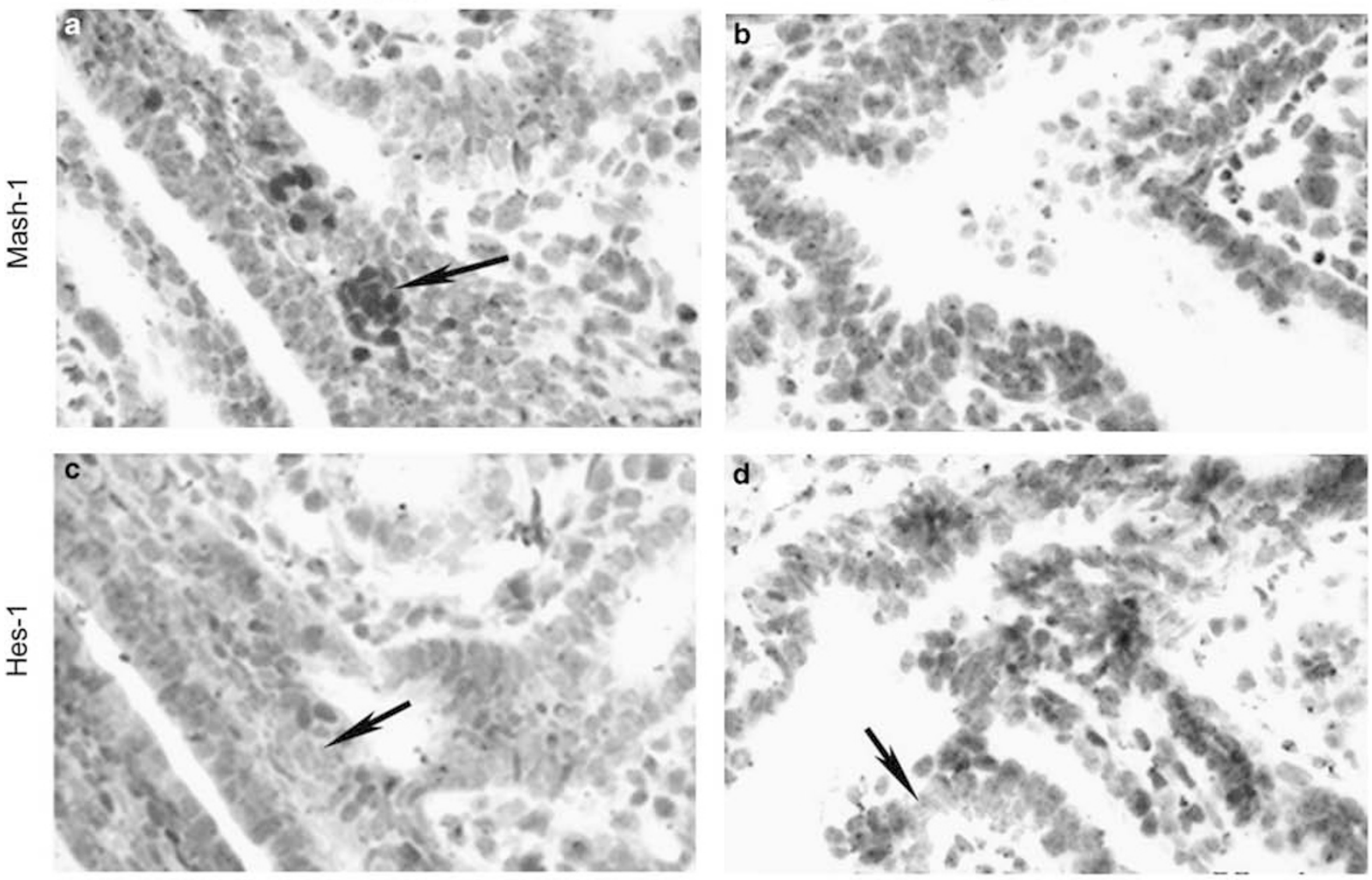

$E 16+6 d$
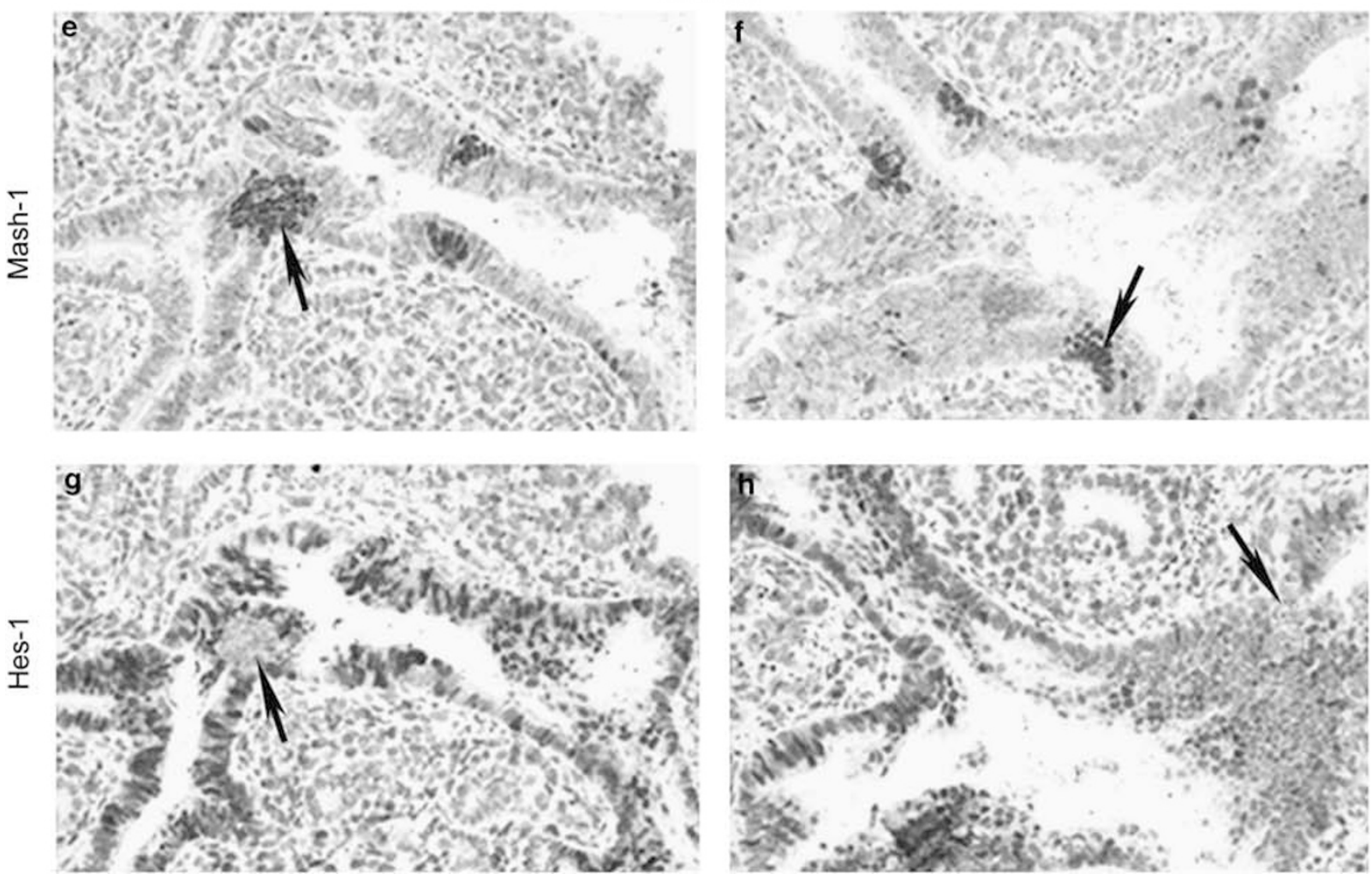
For NEB to become fully functional as airway sensors, they require extensive innervation, derived principally from the nodose ganglion. ${ }^{20}$ We postulate that Prox-1 may be involved in regulating the process of PNEC/NEB innervation. It has been shown recently that Prox- 1 differentially regulates neuropilins, NRP1 and NRP2, involved in axonal guidance and angiogenesis. ${ }^{21}$ Mash-1/Prox-1-positive precursor cells are found throughout the $\mathrm{CNS}^{15}$ and $\mathrm{PNS}^{22}$ and therefore coexpression of these two transcription factors seems to be a general phenomenon during the early stages of neural development. During later stages of development, Prox-1 expression becomes restricted to the hippocampus and cerebellar neurons. ${ }^{16}$ Other studies have linked Prox-1 to an involvement in the subsequent specification of other neural progeny ${ }^{15,23,24}$ in both the CNS and PNS.

Our RT-PCR and western blot analyses of a gestational series of lungs up to P4 clearly show that Mash-1 expression peaks at E15, correlating with the immunostaining for Mash1, whereas Hes-1 expression is relatively constant throughout lung development, with an apparent increase postnatally. Upstream to Mash-1 are other members of the Notch family of neurogenic genes that delineate neuroendocrine $v s$ nonneuroendocrine cell fates. ${ }^{25}$

In the lung, expression of ASCL1 (ASH-1) in humans and Ascl1 (Mash-1) in mice directs PNEC differentiation, whereas Hes-1 expression regulates the differentiation of nonneuroendocrine cells that also express the Notch1 receptor. ${ }^{25}$ Notch-1 is reduced in the lung epithelium of Hes1-null $(-/-)$ mice, whereas Mash-1 is increased. ${ }^{25}$ In Hes-1 $(-/-)$ lungs, precocious development of PNEC is noted at E13, followed by PNEC hyperplasia by E18. Only a sub-population of airway cells differentiate into PNECs, whereas CC10-expressing cells predominate, suggesting that other factors determine lineage fate as well. Overexpression of Notch-1 in a transgenic mouse by targeting Notch-1 to PNECs through the calcitonin promoter induced a marked decrease in PNECs. ${ }^{26}$ Interestingly, expression of the mammalian Notch 1 ligand, Delta-like 1 (Dll 1), correlates with the appearance of PNECs, ${ }^{27}$ whereas Mash-1-deleted lungs also show loss of Dll $1 .^{25}$ This suggests that Mash-1 may regulate Dll 1 expression and this may also affect the development of nonneuroendocrine cells, an interaction that is still unclear. Interestingly, knockdown of Notch-1 expression with antisense oligonucleotides in culture results in increased branching of embryonic lung buds, ${ }^{27}$ as seen here with hypoxia, although we did not observe a change in Hes-1 ex- pression. In addition, as antisense to Notch-1 markedly increases the number of PNECs, it is conceivable that other factors of the 'neural lateral inhibition' pathway exist and are potentially functional in the lung. ${ }^{28}$

Our studies on the effects of hypoxia on PNEC/NEB cell differentiation in the developing lung clearly show that shortterm $(24 \mathrm{~h})$ and sustained, long-term (6 days) hypoxia downregulates Mash-1 expression and leads to loss of the PNEC/NEB phenotype.

We also show that this sensitivity to hypoxia is restricted to early stages of lung development and that, on reoxygenation, Mash-1 expression can be restored. Although here we did not assess proliferation after reoxygenation, it is a consideration for future studies, as there was an apparent burst of Mash-1positive clusters. Although we recognize that our data are derived from in vitro experimental models, it may simulate effects of intermittent hypoxia that are known to be a significant factor in a number of clinical settings. ${ }^{29}$. In other cell systems, hypoxia is known to downregulate the expression of neuronal/neuroendocrine marker genes and to induce neural crest sympathetic progenitors expressing Notch- $1^{30}$ and structural proteins such as nestin associated with the stem cell state. ${ }^{31}$ It can be inferred that Mash-1-positive cells represent an undifferentiated, but perhaps committed, neuroepithelial cell type and that low-oxygen tension maintains the undifferentiated state or, at least, does not allow progression of differentiation. Recent studies shed more light on the possible role of Notch and its relevance to PNEC/NEB and hypoxia. In non-small-cell lung carcinomas, hypoxia elevates Notch signaling and downstream Hes-1 expression, ${ }^{32}$ whereas inducing Notch expression in small-cell lung carcinoma (derived from PNEC) causes cell cycle arrest. ${ }^{33}$ Interestingly, in human embryonic stem cells that are in continuous hypoxia, similar to $5 \% \mathrm{O}_{2}$ used here, Notch activation is maintained and spontaneous differentiation is suppressed, with differentiation rapidly resuming after a switch to normoxia. ${ }^{34}$ Thus, evidence from different embryonic lineages strongly supports the idea that hypoxia keeps progenitors in a more undifferentiated state, which is reversible on increasing oxygenation. Given the idea of cell context-dependent regulatory mechanisms and outcomes, our observations support a central role for hypoxia in the regulation of PNEC/NEB development. Whether this is relevant for PNEC pathobiology still needs to be determined. ${ }^{35}$

Our findings on western blot and RT-PCR analyses of lung organ cultures suggest that hypoxia modulates Mash-1

Figure 6 Effect of gestational age on responsiveness of PNEC/NEB to hypoxia. Comparison of immunohistochemical labeling for Mash-1 and Hes-1 in lung explants from E12 (a-d) and E16 (e-h) grown for 6 days under normoxia or hypoxia. In lung explants from E12 maintained for 6 days under normoxia, both Mash-1 (a) and Hes-1 (c) were strongly expressed. Arrow in panel c points to an NEB that is Hes-1 negative. In similar cultures exposed to hypoxia, NEB lacked expression of Mash-1, (b) but Hes-1 expression remained unaffected (d), as noted by epithelial cells showing positive nuclear staining for Hes-1 surrounding a Hes-1-negative NEB (arrow) (original magnification $\times 600$ ). In contrast, organ cultures from E16 lungs exposed for 6 days to normoxia (e, $\mathbf{g}$ ) or hypoxia ( $\mathbf{f}, \mathbf{h})$ maintained strong expression of both Mash-1 and Hes-1 under either normoxia or hypoxia. Arrows point to NEB cells that are Mash-1 positive and Hes-1 negative. Note that in E16 $+6 \mathrm{~d}$ cultures, the surrounding epithelium is positive for Hes-1 (original magnification $\times 400$ ). 


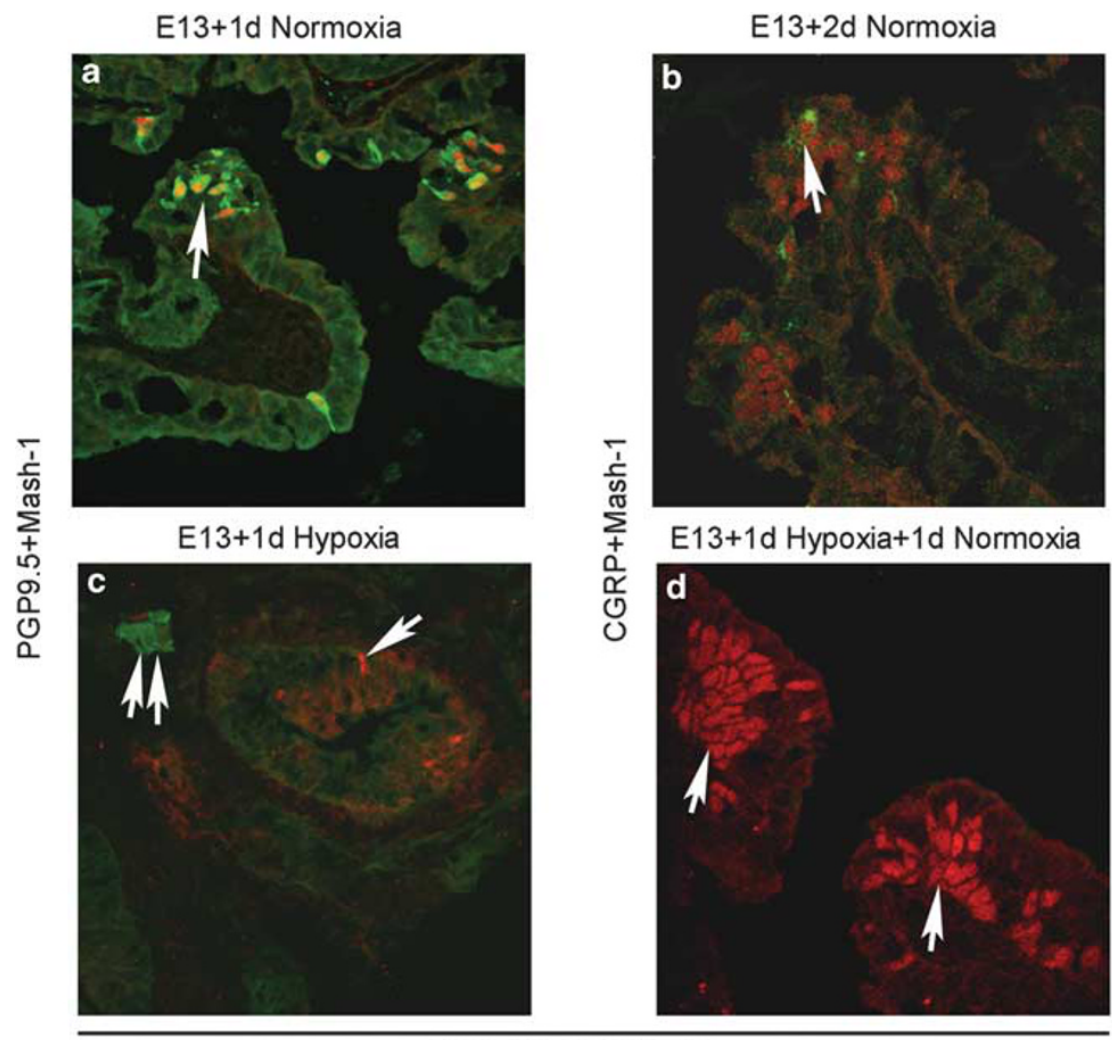

Short Term Cultures

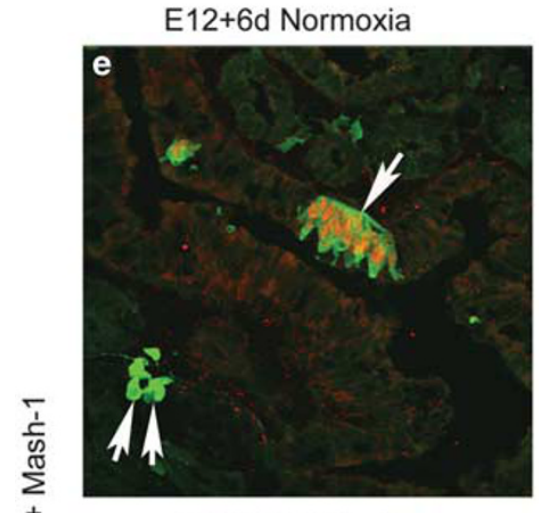

E12+6d Hypoxia

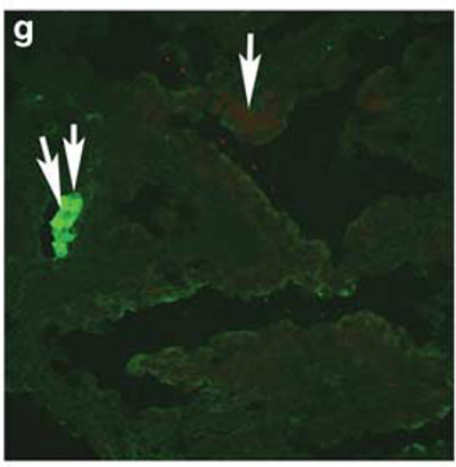

E12+7d Normoxia

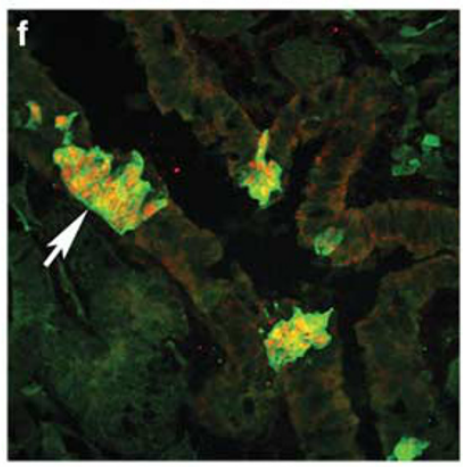

E12+6d Hypoxia+1d Normoxia

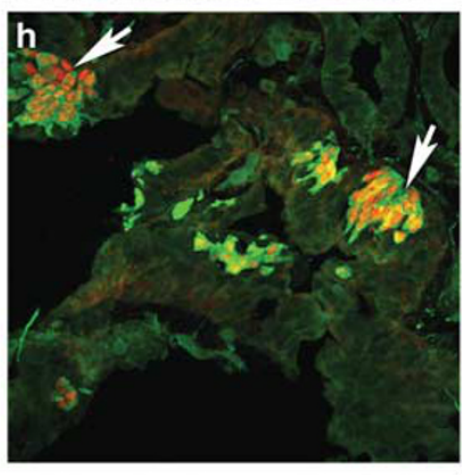

Long term cultures 
expression in the lung, but the precise mechanism is unknown. Hypoxia-inducible factors (HIFs) are known to be intimately involved in cell and tissue responses to $\mathrm{pO}_{2}{ }^{36} \mathrm{HIFs}$ (HIF1-3) are differentially expressed in different cell compartments during lung development, ${ }^{37}$ whereas differentiation of alveolar epithelium requires HIF-1a, likely through regulation of angiogenesis. ${ }^{38}$ Whether HIF regulates the development of PNEC/NEB is yet to be explored.

Taken together, the data present a developmental scenario as follows: During early fetal lung development, Mash-1positive cells start to appear within primitive undifferentiated lung epithelial cells, resembling a primitive neuroepithelium. Although a low-oxygen environment $\left(5 \% \mathrm{O}_{2}\right)$ stimulates pulmonary epithelial branching morphogenesis, it retards PNEC/NEB differentiation but seems to amplify putative PNEC progenitors. Increased epithelial branching is accompanied by increased cell proliferation as shown by BrdU labeling. Van Tuyl et al ${ }^{10}$ explored the effects of hypoxia using a similar in vitro model and found that epithelial differentia- tion was maintained at low oxygen as determined by surfactant protein $\mathrm{C}$ expression. The fact that a fine balance in oxygenation is likely to be obligatory for normal lung development is shown by the fact that hyperoxia has an opposite effect by significantly reducing branching and by inducing apoptosis. ${ }^{39}$ PNEC/NEB commitment is then driven by Mash-1 and maintained under increasing $\mathrm{pO}_{2}$ (toward normoxia) until E15, at which point, Mash-1 regulation becomes $\mathrm{pO}_{2}$ independent. Thus, $\mathrm{E} 15$ seems to be the critical transition point in mouse lung development for maturation of the PNEC/NEB lineage. We therefore predict the existence of a similar critical $\mathrm{pO}_{2}$-dependent gestational threshold in other species.

Finally, the findings reported here could provide important clues to the pathogenesis of several pediatric lung disorders, as well other areas of human pathobiology.

Hyperplasia of PNEC/NEB cells has been described in various developmental and perinatal lung disorders, including pulmonary hypoplasia, bronchopulmonary dysplasia
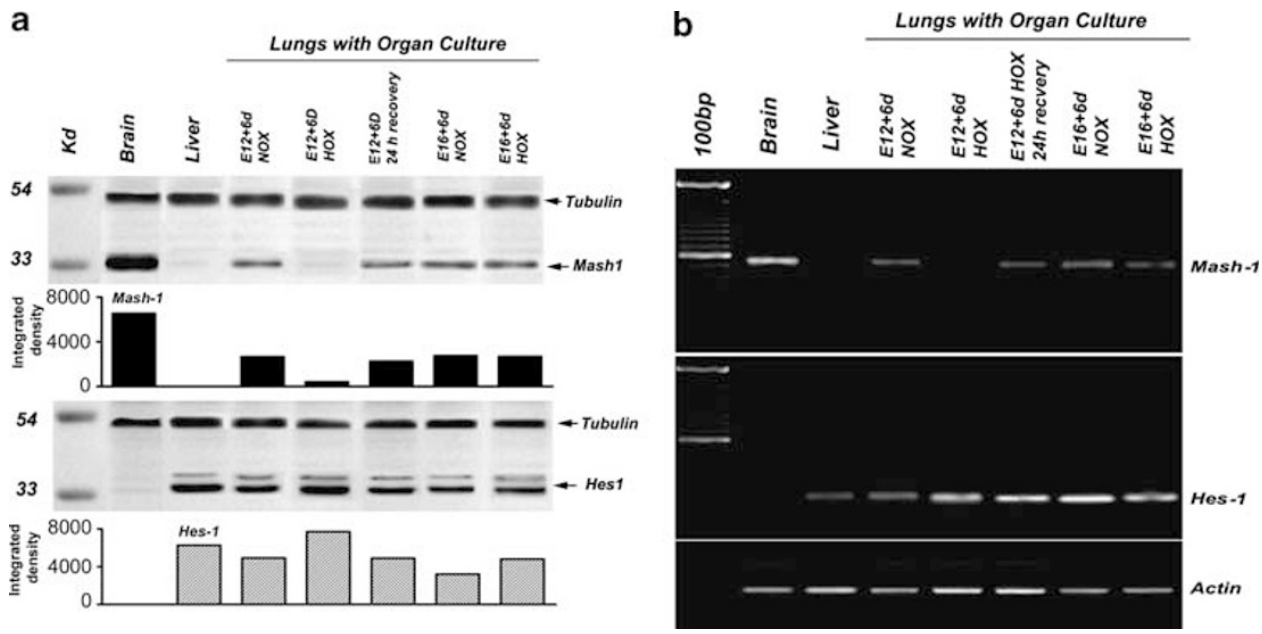

Figure 8 Analysis for expression of Mash-1 and Hes-1 in lung organ cultures. Western blot (a) and RT-PCR (b) were performed on E12 and E16 lungs in organ culture under normoxia and hypoxia. E12 lungs cultured under normoxia express Mash-1, whereas E12 lungs cultured under hypoxia show almost complete loss of Mash-1 expression. However, when these hypoxia-cultured lung explants were returned to normoxia for $24 \mathrm{~h}$, there was a significant upregulation in Mash-1 expression (recovery). In contrast, E16 lungs did not exhibit any loss of Mash-1 expression under hypoxia. In comparison, Hes-1 expression was maintained under all conditions and gestational ages. Densitometric analysis of western blots shown indicates large differences for Mash-1 and some variance for Hes-1, all relative to positive controls for Mash-1 (brain) and Hes-1 (liver). Loading controls are tubulin for western blots and actin for RT-PCR.

Figure 7 Recovery of Mash-1 expression by switching of lung explants from hypoxia to normoxia (reoxygenation) in short-term (1 day, a-d) and long-term (6 day, e-h) cultures. (a) Positive immunoreactivity for PGP9.5 (green signal) and Mash-1 (red signal) in NEB cells (arrow) from E13 lung in normoxia culture for 1 day. (b) Developmental onset of a more mature PNEC/NEB marker CGRP (green signal, arrow), expressed along with Mash-1 (red signal) by day 2 in normoxia culture. (c) In contrast, in parallel organ cultures exposed to 1 day of hypoxia, there is loss of Mash-1 and PGP9.5 expression from NEB cells (arrow), whereas PGP9.5 immunoreactivity in adjacent ganglion cells is preserved (double arrows). (d) When hypoxia cultures are switched back for 1 day of normoxia, there is a robust recovery of Mash-1 expression (arrow) in NEB cell nuclei (red signal); however, note the absence of CGRP expression as compared with (b) (original magnification $\times 630$ ). Long-term organ cultures initiated at E12 showed a strong expression of both PGP9.5 and Mash-1 in NEB cells (arrow) in cultures maintained in normoxia for $6(\mathbf{e})$ or 7 days (f), whereas in organ cultures exposed to hypoxia (g) over the same time period, only very few Mash-1- and no PGP9.5-positive cells are evident. Double arrows indicate positive PGP9.5 staining in ganglia. (h) When these cultures are then switched back to normoxia for 1 day, there is a robust recovery of Mash-1 and PGP9.5 expression in NEB cells (arrow). Parallel control organ cultures in normoxia (f) maintain PGP9.5 and Mash-1 expression in NEB cells (arrow) (original magnification $\times 400$ ). 
(BPD), sudden infant death syndrome (SIDS), and others, but the significance and mechanisms are unknown. ${ }^{40}$ Of particular interest is the so-called 'post surfactant' BPD that occurs in extremely premature infants and is characterized by arrested alveolar development, thickened interalveolar septae, and striking hyperplasia of bombesin/GRP immunoreactive PNEC/NEB in the airways. ${ }^{40}$ It is conceivable that the birth of an extremely premature infant (accustomed to relative intrauterine hypoxic environment) exposes the lungs to higher atmospheric $\mathrm{O}_{2}$ concentration (normoxia), mimicking our in vitro reoxygenation experimental model. In this setting, increased ASH-1 (human homolog of Mash-1) expression could account for PNEC/NEB hyperplasia and, through the effects of increased bombesin/GRP secretion, could lead to interstitial fibrosis and alveolar growth abnormalities. ${ }^{41}$ In the case of SIDS, combined effects of intrauterine intermittent hypoxia and nicotine (in infants whose mothers smoked during pregnancy) could account for NEB cell hyperplasia with a defective chemoreceptor function. ${ }^{42}$ It is also plausible that intrauterine hypoxia/reoxygenation affects the expression of Mash-1 in other organs including the developing CNS and could hence be involved in the pathogenesis of various perinatal neurological disorders. Further studies are clearly indicated to answer these and related questions.

\section{ACKNOWLEDGEMENTS}

The authors greatly appreciate the generosity of $\mathrm{Dr} \mathrm{T}$ Ito (Tokyo) for providing Mash-1-/- tissues, Dr G Oliver for Prox 1-/- tissues, and Dr T Sudo (Toray Industries, Japan) for the Hes-1 antibody. We thank Catherine Luk (Hospital for Sick Children) for helpful assistance with genotyping Prox1 tissue, and Robert Qaqish and Bikul Das (Hospital for Sick Children) for helpful assistance with selection of PCR primers and western blotting, respectively. This study was supported by Canadian Institutes of Health Research grants: \#MOP-12742, MGP 15270 to EC and HY.

\section{DISCLOSURE/CONFLICT OF INTEREST}

The authors declare no conflict of interest.

1. Cutz E, Gillan JE, Bryan AC. Neuroendocrine cells in the developing human lung: morphologic and functional considerations. Pediatr Pulmonol 1985;1:S21-S29.

2. Sunday ME, Hua J, Reyes $B$, et al. Anti-bombesin monoclonal antibodies modulate fetal mouse lung growth and maturation in utero and in organ cultures. Anat Rec 1993;236:25-32.

3. Pan J, Copland I, Post M, et al. Mechanical stretch-induced serotonin release from pulmonary neuroendocrine cells: implications for lung development. Am J Physiol Lung Mol Physiol 2006;290:L185-L193.

4. Sunday ME, Cutz E. The role of neuroendocrine cells in fetal and postnatal lung. In: Mendelson CR (ed). Endocrinology of the Lung. Humana Press: Totowa, NJ, 2000, pp 203-206.

5. Youngson $C$, Nurse $C$, Yeger $H$, et al. Oxygen sensing in airway chemoreceptors. Nature 1993;365:153-156.

6. Wang D, Youngson C, Wong V, et al. NADPH oxidase and hydrogen peroxide-sensitive $\mathrm{K}+$ channel may function as an oxygen sensor complex in airway chemoreceptors and small cell carcinoma cell lines. Proc Natl Acad Sci USA 1996;93:13182-13187.

7. Fu XW, Nurse CA, Wong $V$, et al. Hypoxia-induced secretion of serotonin from intact pulmonary neuroepithelial bodies in neonatal rabbits. J Physiol (London) 2002;539:503-510.
8. Loughna S, Yuan HT, Woolf AS. Effects of oxygen on vascular patterning in the Tie/LacZ metanephric kidney in vitro. Biochem Biophys Res Commun 1998;247:361-366.

9. Yue X, Tomanek RJ. Stimulation of coronary vasculogenesis/ angiogenesis by hypoxia in cultured embryonic hearts. Dev Dyn 1999;216:28-36.

10. Van Tuyl M, Liu J, Wang J, et al. Role of oxygen and vascular development in epithelial branching morphogenesis of the developing mouse lung. Am J Physiol Lung Mol Physiol 2005;288:L167-L178.

11. Johnson JE, Birren SJ, Anderson DJ. Two rat homologues of Drosophila achaete-scute specifically expressed in neural precursors. Nature 1990;346:858-861.

12. Borges MW, Linnoila $\mathrm{Rl}$, van de Velde $\mathrm{HJ}$, et al. An achaete-scute homologue essential for neuroendocrine differentiation in the lung. Nature 1997;386:852-855.

13. Kusakabe $Y$, Miura $H$, Hashimoto $R$, et al. The neural differentiation gene MASH-1 has a distinct pattern of expression from the taste reception-related genes gustducin and T1R2 in the taste buds. Chem Senses 2002;27:445-451.

14. Dyer MA, Livesey FJ, Cepko CL, et al. Prox1 function controls progenitor cell proliferation and horizontal cell genesis in the mammalian retina. Nat Genet 2003;34:53-58.

15. Torii $M$, Matsuzaki F, Osumi N, et al. Transcription factors mash-1 and prox-1 delineate early steps in differentiation of neural stem cells in the developing central nervous system. Development 1999;126: 443-456.

16. Lavado A, Oliver G. Prox1 expression patterns in the developing and adult murine brain. Dev Dyn 2007;236:518-524.

17. Pan J, Yeger H, Cutz E. Innervation of pulmonary neuroendocrine cells and neuroepithelial bodies in developing rabbit lung. J Histochem Cytochem 2004;52:379-389.

18. Sorokin SP, Hoyt Jr RF, Shaffer MJ. Ontogeny of neuroepithelial bodies: correlations with mitogenesis and innervation. Microsc Res Tech 1997;37:43-61.

19. Haley KJ, Drazen JM, Osthanondh R, et al. Comparison of the ontogeny of protein gene product 9.5, chromograninA and nuclear proliferating antigen in developing human lung. Microsc Res Tech 1997;37:62-68.

20. Adriaesen $D$, Timmermans JP, Brouns $L$, et al. Pulmonary intraepithelial vagal nodose afferent nerve terminals are confined to neuroepithelial bodies: an anterograde tracing and confocal microscopy study in adult rats. Cell Tiss Res 1998;293:395-405.

21. Bielenberg DR, Pettaway CA, Takashima S, et al. Neuropilins in neoplasms: expression, regulation and function. Exp Cell Res 2006:312:584-593.

22. Lo LC, Johnson JE, Wuenschell CW, et al. Mammalian achaete-scute homolog 1 is transiently expressed by spatially restricted subsets of early neuroepithelial and neural crest cells. Genes Dev 1991;5: 1524-1537.

23. Doe $C Q$, Technau GM. Identification and cell lineage of individual neural precursors in the Drosophila CNS. Trends Neurosci 1993;16: 510-514.

24. Hirata J, Nagagoshi $H$, Nabeshima $Y$, et al. Assymetric segregation of the homeodomain protein Prospero during Drosophila development. Nature 1995;377:627-630.

25. Ito T, Udaka N, Yazawa T, et al. Basic helix-loop-helix transcription factors regulate the neuroendocrine differentiation of fetal mouse pulmonary epithelium. Development 2000;127:3913-3921.

26. Shan L, Aster JC, Sklar J, et al. Notch-1 regulates pulmonary neuroendocrine cell differentiation in cell lines and transgenic mice. Am J Physiol Lung Cell Mol Physiol 2007;292:L500-L510.

27. Post LC, Ternet M, Hogan BL. Notch/Delta expression in the developing mouse lung. Mech Dev 2000;98:95-98.

28. Kong $Y$, Glickman J, Subramaniam $M$, et al. Functional diversity of notch family genes in fetal lung development. Am J Physiol Lung Cell Mol Physiol 2004;286:L1075-L1083.

29. Prabhakar NR, Fields RD, Baker T, et al. Intermittent hypoxia: cell to system. Am J Physiol Lung Cell Mol Physiol 2001;281: L524-L528.

30. Jogi $\mathrm{A}$, Ora I, Nilsson $\mathrm{H}$, et al. Hypoxia alters gene expression in human neuroblastoma cells toward an immature and neural crest-like phenotype. Proc Natl Acad Sci USA 2002;99:7021-7026. 
31. Wiese C, Rolletschek A, Kania G, et al. Nestin expression - a property of multilineage progenitor cells? Cell Mol Life Sci 2004;61:2510-2522.

32. Chen Y, De Marco MA, Graziani I, et al. Oxygen concentration determines the biological effects of NOTCH-1 signaling in adenocarcinoma of the lung. Cancer Res 2007:67:7054-7059.

33. Sriuranpong V, Borges MW, Ravi RK, et al. Notch signaling induces cell cycle arrest in small cell lung cancer cells. Cancer Res 2001;61:3200-3205.

34. Prasad SM, Czepiel M, Cetinkaya C, et al. Continuous hypoxia culturing maintains activation of Notch and allows long-term propagation of human embryonic stem cells without spontaneous differentiation. Cell Prolif 2009;42:63-74.

35. van Tuyl M, Groenman F, Kuliszewski M, et al. Overexpression of lunatic fringe does not affect epithelial cell differentiation in the developing mouse lung. Am J Physiol Lung Cell Mol Physiol 2005;288:L672-L682.

36. Semenza G. Regulation of oxygen homeostasis by hypoxia-inducible factor 1. Physiology 2009:24:97-106.
37. Rajatapiti P, van der Horst IW, de Rooij JD, et al. Expression of hypoxiainducible factors in normal human lung development. Pediatr Dev Pathol 2008;11:193-199.

38. Saini Y, Harkema JR, LaPres JJ. HIF1alpha is essential for normal intrauterine differentiation of alveolar epithelium and surfactant production in the newborn lung of mice. J Biol Chem 2008;283:33650-33657.

39. Esquibies $A E$, Bazzy-Asaad $A$, Ghassemi $F$, et al. VEGF attenuate hyperoxia injury through decreased apoptosis in explanted rat embryonic lung. Pediatr Res 2008;63:20-25.

40. Cutz E, Yeger H, Pan J. Pulmonary neuroendocrine cell system in pediatric lung disease - recent advances. Pediatr Dev Pathol 2007:10:419-435.

41. Ashour $\mathrm{K}$, Shan L, Lee $\mathrm{JH}$, et al. Bombesin inhibits alveolarization and promotes pulmonary fibrosis in newborn mice. Am J Resp Crit Care Med 2006;173:1377-1385.

42. Cutz E, Perrin DG, Hackman R, et al. Maternal smoking and pulmonary neuroendocrine cells in sudden infant death syndrome. Pediatrics 1996;98:668-672. 